Published in final edited form as:

Nature. 2014 August 28; 512(7515): 431-435. doi:10.1038/nature13375.

\title{
miR-34a Blocks Osteoporosis and Bone Metastasis by Inhibiting Osteoclastogenesis and Tgif2
}

\author{
Jing Y. Krzeszinskia ${ }^{1}$, Wei Wei ${ }^{1}$, HoangDinh Huynh ${ }^{1}$, Zixue Jin ${ }^{1}$, Xunde Wang ${ }^{1}$, Tsung- \\ Cheng Chang ${ }^{2}$, Xian-Jin $\mathrm{Xie}^{3,4}$, Lin $\mathrm{He}^{5}$, Lingegowda S. Mangala ${ }^{6,7}$, Gabriel Lopez- \\ Berestein $^{7,8}$, Anil K. Sood ${ }^{6,7,9}$, Joshua T. Mendell ${ }^{2,3}$, and Yihong Wan ${ }^{1,3}$ \\ ${ }^{1}$ Department of Pharmacology, The University of Texas Southwestern Medical Center, Dallas, \\ Texas 75390, USA \\ ${ }^{2}$ Department of Molecular Biology, The University of Texas Southwestern Medical Center, Dallas, \\ Texas 75390, USA \\ ${ }^{3}$ Simmons Cancer Center, The University of Texas Southwestern Medical Center, Dallas, Texas \\ 75390, USA
}

${ }^{4}$ Department of Clinical Sciences, The University of Texas Southwestern Medical Center, Dallas, Texas 75390, USA

${ }^{5}$ Division of Cellular and Developmental Biology, Molecular and Cell Biology Department, University of California at Berkeley, Berkeley, California 94705, USA

${ }^{6}$ Department of Gynecologic Oncology and Reproductive Medicine, The University of Texas MD Anderson Cancer Center, Houston, Texas 77030, USA

${ }^{7}$ Center for RNA Interference and Non-coding RNA, The University of Texas MD Anderson Cancer Center, Houston, Texas 77030, USA

${ }^{8}$ Department of Experimental Therapeutics, The University of Texas MD Anderson Cancer Center, Houston, Texas 77030, USA

${ }^{9}$ Department of Cancer Biology, The University of Texas MD Anderson Cancer Center, Houston, Texas 77030, USA

\section{Abstract \\ The bone resorbing osteoclasts significantly contribute to osteoporosis and cancer bone metastases ${ }^{1-3}$. MicroRNAs (miRNAs) play important roles in physiology and disease ${ }^{4,5}$, and present tremendous therapeutic potential ${ }^{6}$. Nonetheless, how miRNAs regulate skeletal biology is}

\footnotetext{
Users may view, print, copy, and download text and data-mine the content in such documents, for the purposes of academic research, subject always to the full Conditions of use:http://www.nature.com/authors/editorial_policies/license.html\#terms

Corresponding author: Yihong Wan (yihong.wan@utsouthwestern.edu).

AUTHOR CONTRIBUTIONS

J.Y.K. and Y.W. conceived the project and designed the experiments. All experiments, except the ones listed below, were performed by J.Y.K. W.W. assisted with $\mu$ CT, ELISA and histomorphometry analyses. H.D.H. assisted with bone marrow transplantation and injection. Z.J. assisted with FACS analyses. X.W assisted with western blot analyses. T.C.C. assisted with northern blot analyses and life-span experiments. X.J.X. assisted with statistical analyses. L.H. provided the full miR-34a KO mice. L.S.M., G.L.B. and A.K.S. assisted with nanoparticle packaging. J.T.M. provided the miR-34a gene trap KO mice. Y.W. wrote the manuscript.
} 
underexplored. Here we identify miR-34a as a novel and critical suppressor of osteoclastogenesis, bone resorption and the bone metastatic niche. miR-34a is down-regulated during osteoclast differentiation. Osteoclastic miR-34a over-expressing transgenic mice exhibit lower bone resorption and higher bone mass. Conversely, miR-34a knockout and heterozygous mice exhibit elevated bone resorption and reduced bone mass. Consequently, ovariectomy-induced osteoporosis, as well as bone metastasis of breast and skin cancers, are diminished in osteoclastic miR-34a transgenic mice, and can be effectively attenuated by miR-34a nanoparticle treatment. Mechanistically, we identify Tgif2 (transforming growth factor-beta-induced factor 2) as an essential direct miR-34a target that is pro-osteoclastogenic. Tgif2 deletion reduces bone resorption and abolishes miR-34a regulation. Together, using mouse genetic, pharmacological and disease models, we reveal miR-34a as a key osteoclast suppressor and a potential therapeutic strategy to confer skeletal protection and ameliorate bone metastasis of cancers.

We examined the levels of several cancer-related miRNAs during a time course of bone marrow osteoclastogenesis assay (Fig. 1a). While the expression of an osteoclast marker tartrate-resistant acid phosphatase (TRAP) was rapidly increased by RANKL and further elevated by rosiglitazone $e^{7,8}$ (Fig. 1b), miR-34a was rapidly down-regulated by RANKL and further diminished by rosiglitazone (Fig. 1c). The levels of miR-34b/c, two other members in the miR-34 family, were unaffected and expressed at much lower levels than miR-34a (Fig. 1d).

The sequence of miR-34a is evolutionally conserved and identical in mice and humans. Osteoclast differentiation from both mouse bone marrow precursors (Fig. 1e-f) and human peripheral blood mononuclear cells (hPBMN) (Fig. 1g-j) was inhibited by a miR-34a precursor (pre-miR-34a) but enhanced by an antisense miR-34a inhibitor (anti-miR-34a), indicating that miR-34a regulation of bone resorption in mice will likely translate to human pathophysiology.

We generated osteoclastic miR-34a transgenic mice using CAG34a mice (Fig. 2a) and Tie2cre mice ${ }^{7,8}$. FACS and imaging showed that osteoclast progenitors from the 34a-Tie2-Tg $\left(\mathrm{CAG} 34 \mathrm{a}^{+} \mathrm{Cre}^{+}\right)$mice were converted to $\mathrm{GFP}^{+} \mathrm{LacZ}^{-}$whereas the controls $\left(\mathrm{CAG} 34 \mathrm{a}^{+} \mathrm{Cre}^{-}\right)$ remained GFP-LacZ ${ }^{+}$(Extended Data Fig. 1a-b). Northern blot confirmed the overexpression of mature-miR-34a in the bone marrow of 34a-Tie2-Tg mice (Extended Data Fig. 1c).

Osteoclast differentiation assay reveal that the higher levels of mature miR-34a in the 34aTie2-Tg cultures resulted in a lower induction of osteoclast markers, diminished number/ size of mature osteoclasts, and reduced resorptive activity, whereas precursor proliferation or survival was unaltered (Fig. 2b, Extended Data Fig. 1d-g). Consequently, serum bone resorption marker CTX-1 (C-terminal telopeptides of Type I collagen) and osteoclast number were decreased, whereas osteoblast number, bone formation rate (BFR) and mineral apposition rate (MAR) were unaltered (Fig. 2c, Extended Data Fig. 1h-i).

$\mu \mathrm{CT}$ analysis of the proximal tibiae showed that 34a-Tie2-Tg mice had increased bone mass and decreased structure model index (SMI), which quantifies the relative amount of plates (SMI=0, strong) and rods (SMI=3, fragile) (Fig. 2d-e). Cortical BV/TV was also higher 
(Fig. 2f). Moreover, miR-34a transgenic mice generated by three other osteoclast-targeting cre drivers also exhibited a similar phenotype (Extended Data Fig. 2, 3). Thus, miR-34a in the osteoclast lineage augment bone mass by suppressing osteoclastogenesis and bone resorption.

To determine whether miR-34a is a physiologically relevant regulator of bone resorption, we next examined miR-34a knockout (34a-KO) and heterozygous (34a-Het) mice (Fig. 2g). Northern blot confirmed the diminished levels of miR-34a in 34a-KO (Extended Data Fig. 4a). Consistent with prior reports 9,10 , miR-34a deletion had no overt effect on mouse development. Osteoclast differentiation was augmented in 34a-Het and 34a-KO cultures, while precursor proliferation or survival was unaffected (Fig. 2h, Extended Data Fig. 4be). As a result, serum CTX-1 and osteoclast number were elevated (Fig. 2i, Extended Data Fig. 4g-h). $\mu \mathrm{CT}$ revealed that $34 \mathrm{a}-\mathrm{KO}$ and 34a-Het mice exhibited a low-bone-mass with decreased connectivity density (Conn.D) and increased SMI (Fig. 2j-l). Global miR-34a deletion also decreased bone formation as the serum marker P1NP (N-terminal propeptide of type I procollagen), osteoblast number, BFR and MAR were reduced (Fig. 2m, Extended Data Fig. 4g-h). The increased resorption in 34a-Het indicates that miR-34a function is haploinsufficient and sensitive to dosage reduction. The recently published miR-34abc triple $\mathrm{KO}(34 \mathrm{abc}-\mathrm{TKO})^{10}$ and full miR-34a $\mathrm{KO}^{9}$ also showed a similar phenotype (Extended Data Fig. 5a-h), which validate our miR-34a gene trap mice and strengthen the finding that miR-34a loss-of-function elevates bone resorption.

Bone marrow transplantation showed that WT mice receiving 34a-KO marrow also exhibited higher CTX-1 (Extended Data Fig. 4f) compared to WT mice receiving WT marrow. Furthermore, osteoclastic miR-34a conditional KO mice (34a-Tie2-KO) also exhibited elevated osteoclast differentiation and bone resorption, but unaltered bone formation, leading to a decreased bone mass (Extended Data Fig. 5i-n). Thus, miR-34a deletion in the osteoclast lineage elevates bone resorption.

Our genetic findings prompt us to investigate whether pharmacological administration of a miR-34a mimic can attenuate postmenopausal osteoporosis using an ovariectomy (OVX) mouse model and a chitosan $(\mathrm{CH})$ nanoparticle vehicle. Reduction of uterine weight in all ovariectomized mice indicated effective estrogen depletion (Fig. 3a). Unaltered body weight indicates the absence of obvious toxicity from $\mathrm{CH}$ nanoparticles (Fig. 3b). Compared to sham controls, OVX mice treated with miR-Ctrl-CH showed increased CTX-1 and decreased P1NP, whereas both effects were largely prevented in OVX mice treated with miR-34a-CH (Fig. 3c-d). Consequently, OVX-induced bone loss was attenuated by miR-34a-CH (Fig. 4e-f; Extended Data Fig. 6a). miR-34a-CH also decreased bone resorption and increased bone formation in sham controls, leading to a higher bone mass (Extended Data Fig. 6b-d). Biodistribution analysis showed that miR-34a level in the bone marrow was the highest, and further increased by 5 -fold by miR-34a- $\mathrm{CH}$, indicating an efficient miR-34a delivery (Fig. 3g).

In addition to acute systemic miR-34a treatment, we also examined the effects of chronic osteoclastic miR-34a over-expression. OVX-induced bone resorption and bone loss were also attenuated in 34a-Tie2-Tg mice without altering OVX effects on bone formation (Fig. 
3h-k, Extended Data Fig. 6e). These results indicate that osteoclastic miR-34a overexpression is sufficient to impede osteoporosis, and osteoclast is a key site for miR-34a therapeutic benefit.

To determine if osteoclastic miR-34a confers protection from bone metastases, we employed two cancer-cell-cardiac-injection models. First, a human breast cancer cell line (MDA231BoM-1833) was xenografted into female nude mice. This model allows us to assess cancer cells from human. Second, a mouse melanoma cell line (B16-F10) was allografted into immunocompetent male mice. This model took consideration of adaptive immunity. In both models, bone metastases were attenuated in 34a-Tie2-Tg and 34a-PT-Tg mice but exacerbated in 34a-KO and 34a-Het mice (Fig. 31-o, Extended Data Fig. 7a-f). Since miR-34a remained intact in the exogenous cancer cells, the altered bone metastases resulted from the altered miR-34a in the bone microenvironment of the host.

Pharmacologically, we tested both a treatment protocol using the human breast cancer model and a prevention protocol using the mouse melanoma model. In both cases, bone metastases were diminished by miR-34a-CH (Fig. 3p-s). Systemic miR-34a-CH delivery affected neither tumor growth nor metastasis to other organs such as lung (Extended Data Fig. 8ab). Moreover, treating only the cancer cells with miR-34a-CH before injection had no effect (Extended Data Fig. 8c-d). Consistent with the published finding that miR-34abc deletion does not increase tumorigenesis ${ }^{10}$, our $34 \mathrm{a}-\mathrm{KO}$ mice also showed unaltered cancer susceptibility (Extended Data Fig. 8e).

Since systemic miR-34a-CH treatment not only decreases bone resorption but also increases bone formation, we examined the effects of miR-34a over-expression in osteoblasts. We bred the CAG34a mice with Osterix-CreER mice to generate 34a-Osx-Tg mice. Osteoblast differentiation was reduced for $34 \mathrm{a}-\mathrm{KO}$ and $34 \mathrm{a}-\mathrm{Het}$ mice, but increased for $34 \mathrm{a}-\mathrm{Osx}-\mathrm{Tg}$ mice (Extended Data Fig. 9a-d). Consequently, 34a-Osx-Tg mice exhibited a higher bone formation but unaltered bone resorption, leading to an increased bone mass (Extended Data Fig. 9e-g). Importantly however, the elevated bone formation alone in the 34a-Osx-Tg mice was insufficient to attenuate either OVX-induced bone loss or cancer bone metastases (Extended Data Fig. 9h-i). Together, our conditional miR-34a transgenic mouse models pinpointed the mechanisms underlying the therapeutic benefits of miR-34a by revealing that osteoclast, rather than cancer cell or osteoblast, is the critical and essential player.

To elucidate the mechanisms, we identified Tgif 2 as a novel direct miR-34a target in the osteoclast lineage (Extended Data Fig. 10a-c). Tgif2 expression was suppressed by miR-34a gain-of-function, but increased by miR-34a loss-of-function, in both mouse and human osteoclast cultures (Fig. 4a-b, Extended Data Fig. 10d-e). The miR-34a seed region in Tgif2 3'UTR is evolutionally conserved in mammals (Fig. 4c). Luciferase reporter assay showed that Tgif2 3'UTR is sufficient to confer miR-34a regulation (Fig. 4d-e). Importantly, when the miR-34a seed region in the Tgif $23^{\prime}$ UTR was mutated, miR-34a regulation was abolished (Fig. 4d-e).

Tgif2 expression was increased during WT osteoclast differentiation (Fig. 4b). Tgif2-KO and Tgif2-Het mice had lower bone resorption and higher bone mass (Fig. 4f-h, Extended 
Data Fig. 10f). Tgif2 deletion reduced osteoclast differentiation, and abolished the antiosteoclastogenic effects of miR-34a (Fig. 4i-j). Moreover, Tgif2/miR-34a double knockout mice (DKO) could no longer increase osteoclast differentiation or bone resorption (Fig. 4kl) compared to Tgif2-KO mice. These results indicate that Tgif2 is pro-osteoclastogenic and essential for miR-34a regulation.

We next investigated how Tgif 2 potentiates RANKL signaling. Transfection assays revealed that NFATc1, c-fos and c-jun, also to a lesser extent NFKB (p65), could induce Tgif2 expression (Fig. 4m). Response elements for NFATc1 and AP-1, but not NFkB, were identified in the Tgif 2 promoter region $(-5 \mathrm{~Kb}$ to $+5 \mathrm{~Kb})$. ChIP analysis in osteoclast cultures showed that NFATc1, c-jun and c-fos bound to these sites upon RANKL stimulation, leading to activated Tgif2 transcription shown by the elevated H3K4me3 level at the transcription start site (Fig. 4n). This indicates that NFATc1 and AP-1 induce Tgif2 expression during osteoclastogenesis.

Luciferase reporter assay showed that Tgif 2 augmented the activity of NFATc1, NFKB and c-Jun, but not c-fos (Fig. 6o). Consistently, the activity of endogenous NFATc1, NFKB and c-Jun, but not c-fos, was reduced in Tgif2-KO cultures and enhanced in 34a-KO cultures

(Fig. 4p). Furthermore, NFATc1 mRNA, c-Jun phosphorylation and I $\kappa$ Ba degradation were decreased in Tgif2-KO cultures and increased in 34a-KO cultures (Fig. 4q-r). Therefore, Tgif2 potentiates osteoclastogenesis via a positive feedback loop in which RANKL-induced transcription factors activate Tgif 2 expression, and Tgif 2 in turn promotes their activity. Collectively, these findings reveal Tgif 2 as a novel yet critical regulator of osteoclastogenesis and bone resorption, as well as a key miR-34a direct target that is essential for miR-34a regulation (Fig. 4s).

The roles of miRNAs in bone physiology have just begun to emerge. Recent studies show that osteoblast-specific gain-of-function of miR-34b/c decreases bone mass by suppressing osteoblastogenesis and bone formation ${ }^{11,12}$. Here we show that osteoclast-specific miR-34a gain-of-function protects bone by suppressing osteoclastogenesis and bone resorption. These findings uncover an interesting functional divergence among the miR-34 family members. Our study paves the road for future discovery of other miRNAs that may be regulated by RANKL and/or control Tgif 2 expression, as well as future epidemiological and clinical studies to explore the pathological and therapeutic roles of this miR-34a-Tgif2 pathway in human.

Most systemically delivered drugs can target multiple tissues and cell types. We found that miR-34a also enhances bone formation; although miR-34a over-expression in osteoblast is neither sufficient nor essential for the therapeutic benefits of miR-34a in osteoporosis or bone metastasis, miR-34a may be a superior new therapy that exerts both anti-catabolic and anabolic effects compared to the current drugs that are solely anti-catabolic. Our identification of miR-34a, as well as the recent report of miR-141 and miR-21913, opens an exciting avenue for the development of a whole new generation of RNA-based osteoprotective medicine. 
Other miR-34a target genes have been reported in different biological context, such as SIRT $1{ }^{14}$, SIRT6 ${ }^{15}$ and PNUTS ${ }^{16}$. Although miR-34a may also target genes other than Tgif2 in osteoclasts, our genetic rescue ex vivo and in vivo show that Tgif2 is the key miR-34a target, suggesting that other genes are likely secondary or functionally irrelevant to osteoclastogenesis.

miR-34abc are commonly deleted in human cancers ${ }^{17}$. In vitro studies suggest that miR-34abc may be critical mediators of p53 function and potential tumor suppressors ${ }^{18}$. Surprisingly, in vivo studies reveal that miR-34abc triple KO mice exhibit intact p53 function without increased tumorigenesis ${ }^{10}$. Nonetheless, systemic miR-34a administration can indeed attenuate cancer malignancy ${ }^{19}$. This raises the intriguing possibility that its anticancer effects may reside in other cells that constitute the tumor microenvironment such as the osteoclasts in the bone metastatic niche. Indeed, our findings illustrate that bone metastases are effectively blocked by miR-34a in osteoclasts, thus providing the first in vivo genetic evidence that miR-34a opposes malignant progression of cancer by disarming the metastatic niche.

\section{METHODS SUMMARY}

Conditional miR-34a transgenic mice were generated using the CAG-Z-EGFP vector. miR-34a knockout mice were generated using a gene-trap ES cell line.

\section{METHODS}

\section{Mice}

To generate cre-flox controlled conditional miR-34a transgenic mice (CAG34a), a 431 basepair genomic sequence containing 168 base pairs $5^{\prime}$ and 161 base pairs $3^{\prime}$ of the pre-miR-34a sequence was inserted into the CAG-Z-EGFP vector ${ }^{20}$. Transgenic founders on pure C57BL/6J background were established by pronuclear injection at the UT Southwestern transgenic core. From 14 founders that carry the LacZ and GFP transgenes, we selected 6 founders that had the highest tail lacZ expression, and bred them to cre transgenic mice. Representative results from at least two independent founders are reported here. To establish osteoclastic miR-34a transgenic mice, CAG34a mice were bred with the previously described Tie2cre mice ${ }^{7,8}$, PPAR $\gamma$-tTA;TRE-cre (PT-cre) mice ${ }^{21}$, lysozyme-cre (Lys-cre) mice $^{22}$ or Ctsk-cre mice ${ }^{23}$. To establish osteoblastic miR-34a transgenic mice, CAG34a mice were bred with the previously described Osx-CreER mice ${ }^{24}$. All conditional miR-34a transgenic mice were on pure C57BL/6J background, and compared to littermate controls that carry only the transgene allele or only the Cre allele; representative results for "transgene only" group is shown as "Ctrl" group; consistent with previous studies, these Cre lines alone do not exhibit bone phenotype. miR-34a knockout mice in a C57BL/6-129P2 mixed genetic background were generated using a mouse embryonic stem cell line (International Gene Trap Consortium clone YHA350) harboring a gene-trap integration in the miR-34a transcription unit, and backcrossed to C57BL/6J mice for at least five generations. In this gene-trap allele, a splice-acceptor followed by a $\beta$-geo cassette (fusion of $\beta$-galactosidase and neomycin transferase) was inserted between exon 2 and 3 of the mouse miR-34a gene, leading to a truncated and non-functional pri-miR-34a transcript. miR-34abc 
triple knockout mice and WT controls in a C57BL/6-129SvJae mixed background were provided by Dr. Andrea Ventura (Memorial Sloan-Kettering Cancer Center) ${ }^{10}$. miR-34a full knockout mice and WT controls on a pure C57BL/6 background were provided by Dr. Lin $\mathrm{He}$ (University of California at Berkeley) ${ }^{9}$. Tgif2-KO mice on a C57BL/6-129 mixed background were provided by Dr. David Wotton (University Of Virginia) ${ }^{25}$. miR-34a flox mice on a pure C57BL/6 background were from Jackson Laboratory ${ }^{9}$. Osteoclastic miR-34a conditional KO mice (34a-Tie2-KO) were generated by breeding miR-34a flox mice with Tie2-cre mice. Bone marrow transplantation was performed as described ${ }^{7}$. Briefly, bone marrow cells from 2-month-old male donor (WT or 34a-KO) were intravenously transplanted into five 2-month-old male C57BL/6J recipients that were irradiated at lethal dose (1000 roentgen); the mice were analyzed 3 month post transplantation. Ovariectomy or sham operation was performed on 10-20 week old female mice. miRNA-carrying chitosan $(\mathrm{CH})$ nanoparticles ${ }^{26}$ were delivered by intravenous injections at $5 \mu \mathrm{g} / \mathrm{mouse}$ or $10 \mu \mathrm{g} / \mathrm{mouse}$ twice per week for 4-5 weeks. Sample size estimate was based on power analyses performed using SAS 9.3 TS X64_7PRO platform. With the observed group differences which is of great biological value, and the relatively small variation of the in vivo measurements, a sample size of four per group $(\mathrm{n}=4)$ will provide higher than $90 \%$ power at type I error rate of 0.05 (two-sided test), and a sample size of three per group ( $\mathrm{n}=3$ ) will provide higher than $80 \%$ power at type I error rate of 0.05 (two-sided test). For example, on BV/TV measures with a mean difference of 0.12 between the WT and mutant groups (standard deviation of 0.035 and 0.03 for each of the two groups), 4 mice per group will yield $98 \%$ power and 3 mice per group will yield $83 \%$ power using two sample t-test. Samples were randomly allocated to each group. Analyses were conducted in a blind fashion to the operator. All experiments were conducted using littermates. All protocols for mouse experiments were approved by the Institutional Animal Care and Use Committee of University of Texas Southwestern Medical Center.

\section{Reagents}

Mouse Tgif2 siRNA or control siRNA were from Santa Cruz Biotechnology. miR-34a precursor (pre-miR-34a) and negative control (pre-control), miR-34a inhibitor (antimiR-34a) and negative control (anti-control) were from Life Technologies. All miRNA and siRNA were transfected with Lipofectamine ${ }^{\mathrm{TM}}$ RNAiMAX (Life Technologies) into bone marrow osteoclast progenitors. For in vivo miRNA delivery, HPLC-purified mirVana ${ }^{\mathrm{TM}}$ miR-34a mimic or negative control (Life Technologies) was packaged into chitosan nanoparticles as described ${ }^{26}$. RAW264.7 mouse macrophage cell line was from ATCC (TIB-71). Anti-Tgif2 antibody (catalog \# 09-718) was from Millipore; anti-NFATc1 (7A6), anti c-Jun (H-79) anti IкBa (C-21) antibodies were from Santa Cruz Biotechnologies; antiH3K4me3 (ab8580) and anti-c-fos (ab7963) antibodies were from Abcam; anti-p-c-Jun (Ser73) (catalog \# 9164) was from Cell Signaling.

\section{Bone Analyses}

Micro-Computed Tomography $(\mu \mathrm{CT})$ was performed to evaluate bone volume and architecture using a Scanco $\mu$ CT-35 instrument (SCANCO Medical) as described ${ }^{8}$. Mouse tibiae were fixed in $70 \%$ ethanol and scanned at several resolutions for both overall tibial assessment (14 micron resolution) and the structural analysis of trabecular and cortical bone 
(7 micron resolution). Trabecular bone parameters were calculated using the Scanco software to analyze the bone scans from the trabecular region directly distal to the proximal tibial growth plate. As a bone resorption marker, serum CTX-1 was measured with the RatLaps $^{\text {TM }}$ EIA kit (Immunodiagnostic Systems) ${ }^{27}$. As a bone formation marker, serum amino-terminal propeptide of type I collagen (P1NP) was measured with the Rat/Mouse P1NP enzyme immunoassay kit (Immunodiagnostic Systems) ${ }^{27}$. Static and dynamic histomorphometry were performed with femurs and vertebrae as described ${ }^{27}$. Calcein $(20 \mathrm{mg} / \mathrm{kg}$ ) were injected into 2 month old mice 2 and 10 days before bone collection.

\section{Ex Vivo Osteoclast and Osteoblast Differentiation}

Osteoclasts were differentiated from bone marrow cells as described ${ }^{7,8}$. Briefly, hematopoietic bone marrow cells were purified with $40 \mu \mathrm{m}$ cell strainer, and differentiated with $40 \mathrm{ng} / \mathrm{ml}$ of mouse M-CSF (R\&D Systems) in a-MEM containing 10\% FBS for 3 days, then with 40ng/ml of mouse MCSF and 100ng/ml of mouse RANKL (R\&D Systems) for 3-9 days, in the presence or absence of rosiglitazone $(1 \mu \mathrm{M})$. Mature osteoclasts were identified as multinucleated ( $>3$ nuclei) $\mathrm{TRAP}^{+}$cells. Osteoclast differentiation was quantified by the RNA expression of osteoclast marker genes using RT-QPCR analysis, as well as number and size of mature osteoclasts. For osteoclast resorptive function analyses, bone marrow osteoclast differentiation was conducted in OsteoAssay bone plates (Lonza), and osteoclast activity was quantified as calcium release from bone into culture medium using CalciFluo ELISA assay (Lonza). Osteoclast precursor proliferation was quantified using a bromodeoxyuridine (BrdU) cell proliferation assay kit (GE Healthcare) as described ${ }^{27}$. Osteoclast apoptosis was quantified using Annexin V: PE Apoptosis Detection Kit I (BD Biosciences). Human PBMN cells (ReachBio) were differentiated into osteoclasts in a-MEM containing $10 \%$ FBS, 25ng/ml MCSF, 50ng/ml hRANKL, $1 \mu \mathrm{M}$ Dexamethasome and $1 \mu \mathrm{M}$ rosiglitazone for 14 days; pre-miR or anti-miR were transfected on day 0 and day 6; human RANKL was added on day 7. Osteoblasts were differentiated from bone marrow cells as described ${ }^{27}$.

\section{Gene Expression Analyses}

For mRNA expression, RNA was reverse transcribed into cDNA using an ABI High Capacity cDNA RT Kit (Life Technologies) and then analyzed using real-time quantitative PCR (SYBR Green) in triplicate. All mRNA expression was normalized by L19. For mature miRNA expression, RNA was reverse transcribed into cDNA using NCode VILO miRNA cDNA Synthesis Kit (Life Technologies) and then analyzed in triplicate using real-time quantitative PCR (SYBR Green) and a primer specific for the mature miRNA. All miRNA expression was normalized by sno251.

\section{Identification of miR-34a Targets in the Osteoclast Lineage}

To elucidate the molecular mechanisms for miR-34a inhibition of osteoclastogenessi and bone resorption, we identified key direct miR-34a target genes that are pro-osteoclastogenic. First, we used the TargetScan bioinformatic tool to predict all the miR-34a targets by searching for conserved 8 mer or 7 mer sites that match the miR-34a seed region. Second, we searched databases such as BioGPS to select secondary targets that are expressed in the macrophage-osteoclast lineage. Third, we performed RT-QPCR to select tertiary targets that 
can be inhibited by miR-34a during osteoclast differentiation. Fourth, we performed luciferase reporter assay to test if the $3^{\prime} \mathrm{UTR}$ of each tertiary target could directly suppress gene expression in response to miR-34a. To generate a CMV-Luc-3'UTR reporter, a $\sim 300 \mathrm{bp}$ Tgif2 3'UTR region centering the miR-34a target sequence was cloned into the pMIRREPORT $^{\mathrm{TM}}$ vector (Life Technologies) downstream of the luciferase open reading frame. To generate a mutant reporter with miss-matched miR-34a binding site, the miR-34a target sequence was altered using QuikChange II XL site-directed mutagenesis kit (Stratagene). The reporters were co-transfected with CMV- $\beta$-gal (as an internal transfection control), together with pre-miR-34a or pre-miR-control, anti-miR-34a or anti-miR-control using FuGENE HD reagent (Roche). The transfection assay was conducted in human embryonic kidney 293 cells and CV-1 monkey kidney cells to assess the intrinsic properties of the 3'UTR in different cellular context, and representative results for 293 cells are shown. Luciferase activity was normalized by $\beta$-gal activity.

\section{Bone Metastasis Analyses}

Using a VisualSonics Vevo770 small animal ultrasound device, luciferase-labeled cancer cells were injected into the left cardiac ventricle so that they can bypass the lung and efficiently migrate to the bone ${ }^{28}$. Bone metastases were detected and quantified weekly post injection by bioluminescence imaging (BLI) using a Caliper Xenogen Spectrum instrument at UTSW small animal imaging core facility. The osteolytic metastatic lesions were imaged by radiography using Faxitron Cabinet X-ray System with the X-ray tube voltage fixed at 26 $\mathrm{kVp}$ and the exposure time at $15 \mathrm{~s}$. The luciferase-labeled bone-metastasis-prone MDAMB-231 human breast cancer cell sub-line (MDA231-BoM-1833) ${ }^{29}$ was generously provided by Joan Massagué (a Howard Hughes Medical Institute Investigator at Memorial Sloan-Kettering Cancer Center) and injected into 6-week-old female nude mice (NCI) at $1 \times 10^{5}$ cells/mouse in $100 \mu$ l PBS. The luciferase-labeled B16-F10 mouse melanoma cell line $\mathrm{e}^{30}$ was generously provided by Katherine Weilbaecher (Washington University) and injected into 8-week-old male C57BL/6J mice at $5 \times 10^{4} /$ mouse in $100 \mu \mathrm{l} \mathrm{PBS}$.

\section{Statistical Analyses}

All statistical analyses were performed with Student's t-Test and represented as mean \pm standard deviation (SD) unless noted otherwise. No animal or sample was excluded from the analysis. The $\mathrm{p}$ values were designated as: $*, \mathrm{p}<0.05 ; * *, \mathrm{p}<0.01 ; * * *, \mathrm{p}<0.005 ; * * * *$, $\mathrm{p}<0.001$; n.s. non-significant ( $\mathrm{p}>0.05)$.

\section{ACKNOWLEDGEMENTS}

\footnotetext{
We thank UT Southwestern transgenic core and small animal imaging core for their assistance in our studies; Drs. Paul Dechow, Jerry Feng and Chunlin Qin (Baylor College of Dentistry) for assistance with $\mu \mathrm{CT}$, histomorphometry and X-ray analysis; Dr. Andrea Ventura (Memorial Sloan-Kettering Cancer Center) for miR-34abc triple KO mice; Dr. David Wotton (University Of Virginia) for Tgif2-KO mice; Dr. Hank Kronenberg (Harvard Medical School) for Osx-CreER mice. Dr. Yuji Mishina (University of Michigan) for CAG-Z-EGFP vector. Y. Wan is a Virginia Murchison Linthicum Scholar in Medical Research. This work was in part supported by CPRIT (RP130145, YW; R1008, JM), DOD (BC122877, YW), NIH (R01 DK089113, YW; R01 CA120185 and P01 CA134292, JM; U54 CA151668 and UH2 TR000943, AS; R01 CA139067, LH), The Welch Foundation (I-1751, YW) and UTSW Endowed Scholar Startup Fund (YW). The UTSW Small Animal Imaging Resource is supported in part by the Harold C. Simmons Cancer Center through an NCI Cancer Center Support Grant (1P30 CA142543) and The Department of Radiology. The VisualSonics Vevo 770 was purchased with NIH ARRA stimulus funds 1S10RR02564801. The authors declare that they have no financial conflict of interest.
} 


\section{REFERENCES}

1. Coleman RE. Bone cancer in 2011: Prevention and treatment of bone metastases. Nature reviews. Clinical oncology. 2012; 9:76-78. [PubMed: 22182971]

2. Ell B, Kang Y. SnapShot: Bone Metastasis. Cell. 2012; 151:690-690. e691. [PubMed: 23101634]

3. Novack DV, Teitelbaum SL. The osteoclast: friend or foe? Annu Rev Pathol. 2008; 3:457-484. [PubMed: 18039135]

4. Chivukula RR, Mendell JT. Circular reasoning: microRNAs and cell-cycle control. Trends Biochem Sci. 2008; 33:474-481. [PubMed: 18774719]

5. Ventura A, Jacks T. MicroRNAs and cancer: short RNAs go a long way. Cell. 2009; 136:586-591. [PubMed: 19239879]

6. Kasinski AL, Slack FJ. Epigenetics and genetics. MicroRNAs en route to the clinic: progress in validating and targeting microRNAs for cancer therapy. Nat Rev Cancer. 2011; 11:849-864. [PubMed: 22113163]

7. Wan Y, Chong LW, Evans RM. PPAR-gamma regulates osteoclastogenesis in mice. Nat Med. 2007; 13:1496-1503. [PubMed: 18059282]

8. Wei W, et al. PGC1beta mediates PPARgamma activation of osteoclastogenesis and rosiglitazoneinduced bone loss. Cell Metab. 2010; 11:503-516. [PubMed: 20519122]

9. Choi YJ, et al. miR-34 miRNAs provide a barrier for somatic cell reprogramming. Nature cell biology. 2011; 13:1353-1360. [PubMed: 22020437]

10. Concepcion CP, et al. Intact p53-dependent responses in miR-34-deficient mice. PLoS Genet. 2012; 8:e1002797. [PubMed: 22844244]

11. Bae Y, et al. miRNA-34c regulates Notch signaling during bone development. Hum Mol Genet. 2012; 21:2991-3000. [PubMed: 22498974]

12. Wei J, et al. miR-34s inhibit osteoblast proliferation and differentiation in the mouse by targeting SATB2. The Journal of cell biology. 2012; 197:509-521. [PubMed: 22564414]

13. Ell B, et al. Tumor-induced osteoclast miRNA changes as regulators and biomarkers of osteolytic bone metastasis. Cancer Cell. 2013; 24:542-556. [PubMed: 24135284]

14. Yamakuchi M, Ferlito M, Lowenstein CJ. miR-34a repression of SIRT1 regulates apoptosis. Proceedings of the National Academy of Sciences of the United States of America. 2008; 105:13421-13426. [PubMed: 18755897]

15. Lefort $\mathrm{K}$, et al. A miR-34a-SIRT6 axis in the squamous cell differentiation network. The EMBO journal. 2013; 32:2248-2263. [PubMed: 23860128]

16. Boon RA, et al. MicroRNA-34a regulates cardiac ageing and function. Nature. 2013; 495:107-110. [PubMed: 23426265]

17. Lodygin D, et al. Inactivation of miR-34a by aberrant $\mathrm{CpG}$ methylation in multiple types of cancer. Cell Cycle. 2008; 7:2591-2600. [PubMed: 18719384]

18. Hermeking H. p53 enters the microRNA world. Cancer Cell. 2007; 12:414-418. [PubMed: 17996645]

19. Liu C, et al. The microRNA miR-34a inhibits prostate cancer stem cells and metastasis by directly repressing CD44. Nature medicine. 2011; 17:211-215.

20. Fukuda T, et al. Generation of a mouse with conditionally activated signaling through the BMP receptor, ALK2. Genesis. 2006; 44:159-167. [PubMed: 16604518]

21. Wei $\mathrm{W}$, et al. Osteoclast progenitors reside in the peroxisome proliferator-activated receptor gamma-expressing bone marrow cell population. Mol Cell Biol. 2011; 31:4692-4705. [PubMed: 21947280]

22. Clausen BE, Burkhardt C, Reith W, Renkawitz R, Forster I. Conditional gene targeting in macrophages and granulocytes using LysMcre mice. Transgenic Res. 1999; 8:265-277. [PubMed: 10621974]

23. Nakamura T, et al. Estrogen prevents bone loss via estrogen receptor alpha and induction of Fas ligand in osteoclasts. Cell. 2007; 130:811-823. [PubMed: 17803905] 
24. Maes C, et al. Osteoblast precursors, but not mature osteoblasts, move into developing and fractured bones along with invading blood vessels. Dev Cell. 2010; 19:329-344. [PubMed: 20708594]

25. Powers SE, et al. Tgif1 and Tgif 2 regulate Nodal signaling and are required for gastrulation. Development. 2010; 137:249-259. [PubMed: 20040491]

26. Lu C, et al. Regulation of tumor angiogenesis by EZH2. Cancer Cell. 2010; 18:185-197. [PubMed: 20708159]

27. Wei W, et al. Biphasic and dosage-dependent regulation of osteoclastogenesis by beta-catenin. Mol Cell Biol. 2011; 31:4706-4719. [PubMed: 21876000]

28. Lu X, et al. ADAMTS1 and MMP1 proteolytically engage EGF-like ligands in an osteolytic signaling cascade for bone metastasis. Genes Dev. 2009; 23:1882-1894. [PubMed: 19608765]

29. Kang Y, et al. A multigenic program mediating breast cancer metastasis to bone. Cancer Cell. 2003; 3:537-549. [PubMed: 12842083]

30. Uluckan O, et al. APT102, a novel adpase, cooperates with aspirin to disrupt bone metastasis in mice. Journal of cellular biochemistry. 2008; 104:1311-1323. [PubMed: 18260128] 
a
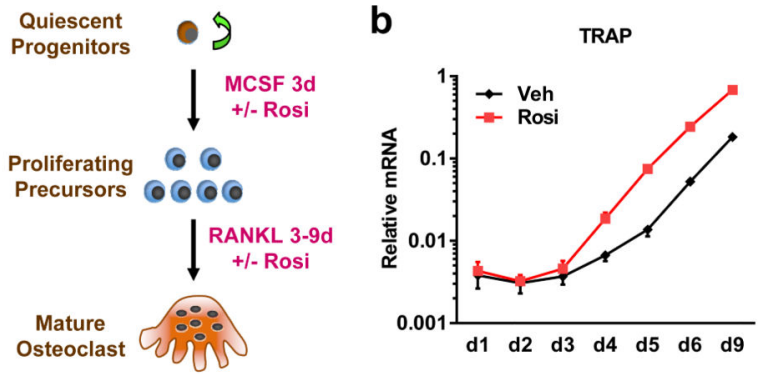

C

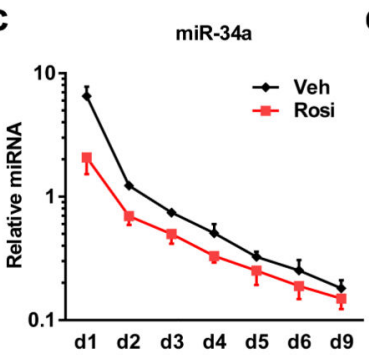

d
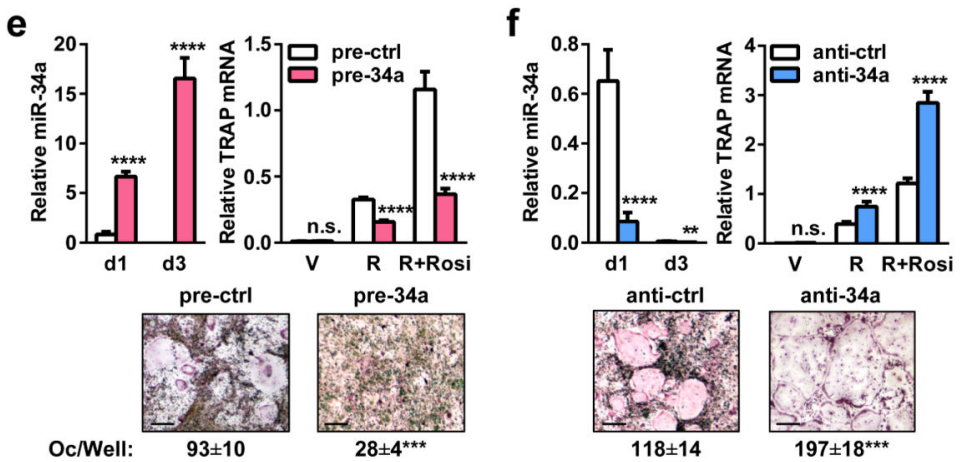

Oc/Well:
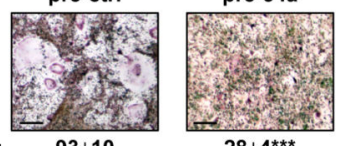

Calcium $(\mu \mathrm{M}): 0.20 \pm 0.02$

$28 \pm 4^{\star \star \star}$
$0.09 \pm 0.01^{*}$

$0.41 \pm 0.03^{*}$
9 hPBMN + hRANKL

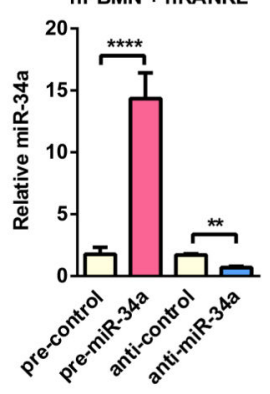

h

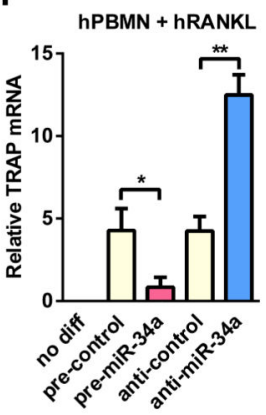

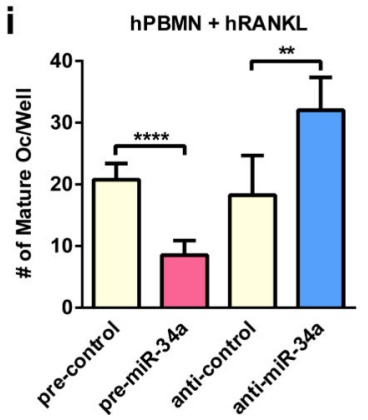

j

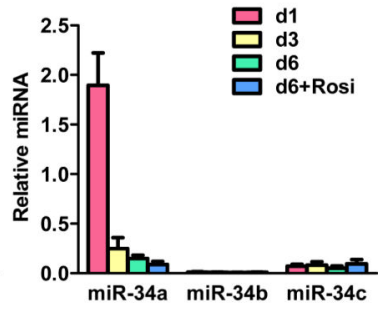

Figure 1. miR-34a Suppresses Osteoclastogenesis Ex Vivo

a, A diagram of bone marrow osteoclast differentiation assay. Rosi, rosiglitazone. b-d, TRAP expression (b) and mature miRNA levels $(\mathbf{c}-\mathbf{d})(\mathrm{n}=3)$. e-f, Osteoclast differentiation was decreased by pre-miR-34a (e) but increased by anti-miR-34a (f) $(n=3)$. Left, mature miR-34a levels; Right, TRAP expression; Bottom, images of TRAP-stained cultures; mature osteoclast numbers (black) and resorptive activity (blue). Scale bar, $25 \mu \mathrm{m}$. R, RANKL. g-j, Human RANKL-mediated osteoclast differentiation from human peripheral blood mononuclear (hPBMN) cells ( $\mathrm{n}=4)$. g, Mature miR-34a levels. h, TRAP expression. i. Mature osteoclast numbers. j. TRAP-staining and resorptive activity. Scale bar, $25 \mu \mathrm{m}$. Error bars, SD. 

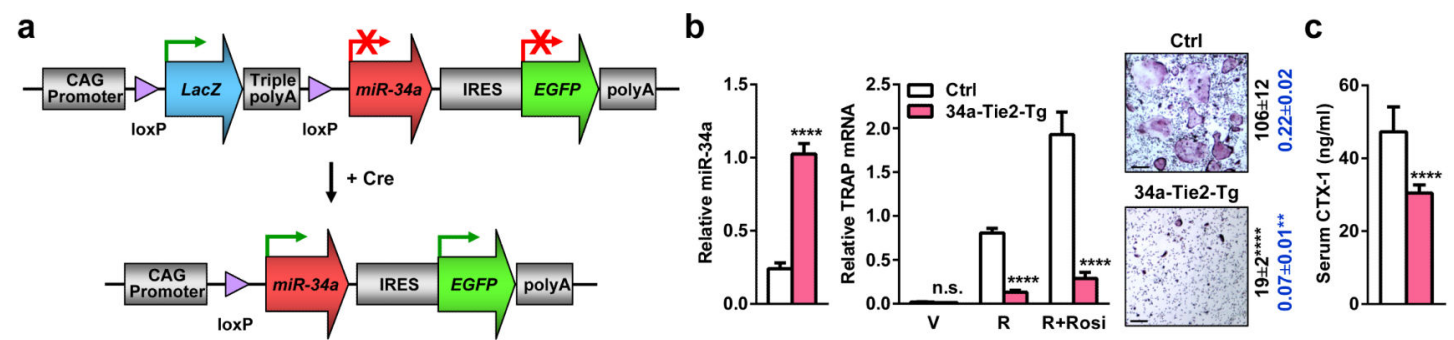

d
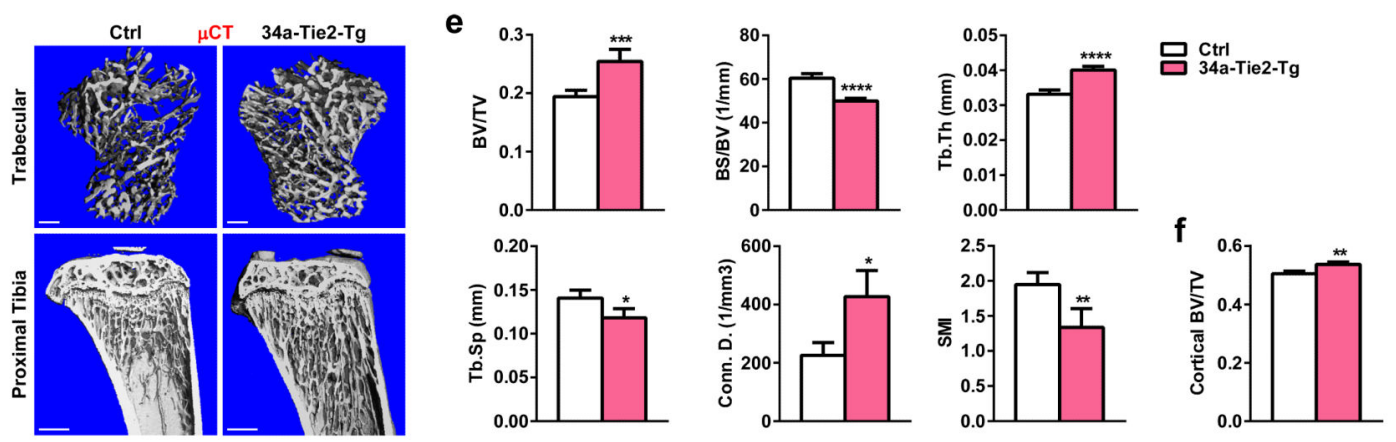

g
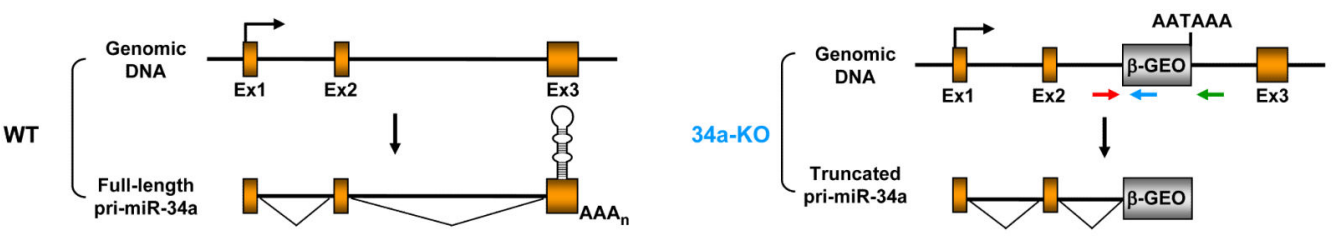

h
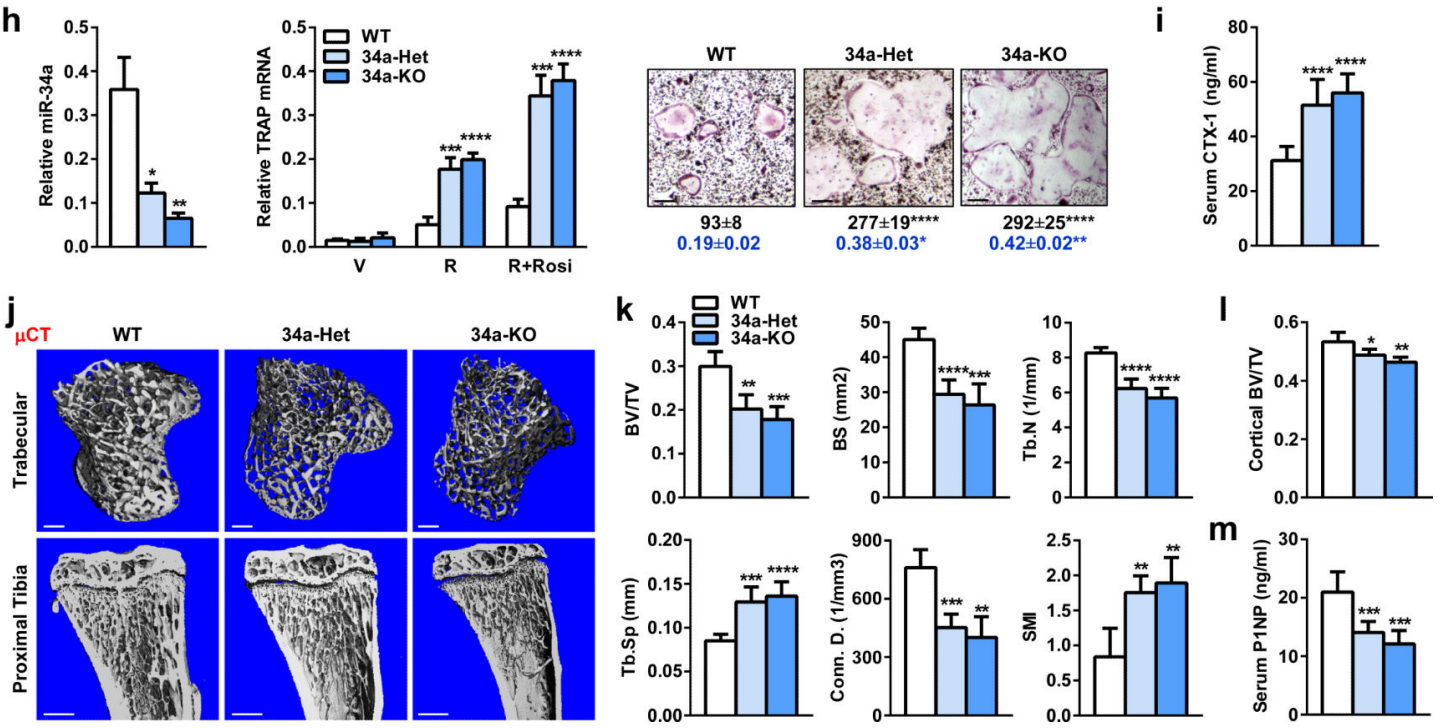

Figure 2. miR-34a Inhibits Bone Resorption and Increases Bone Mass In Vivo

a, A diagram of the conditional miR-34a transgene (CAG-34a). b, 34a-Tie2-Tg cultures showed decreased osteoclast differentiation ( $n=3$ ). Left, miR-34a levels; Middle, TRAP expression; Right, TRAP staining, osteoclast numbers (black) and resorptive activity (blue). Scale bar, $25 \mu \mathrm{m}$. c, Serum CTX-1 (2-month-old, male, $n=5)$. d-f, $\mu \mathrm{CT}$ of the tibiae (2month-old, male, $n=4$ ). $\mathbf{d}$, Images of the trabecular bone of the tibial metaphysis (top) (scale

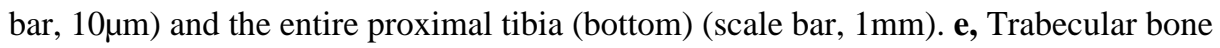
parameters. BV/TV, bone volume/tissue volume ratio; BS/BV, bone surface/bone volume 
ratio; Tb.Th, trabecular thickness; Tb.Sp, trabecular separation; Conn.D., connectivity density; SMI, structure model index. f, Cortical BV/TV. g, A diagram of miR-34a gene-trap knockout. h, 34a-KO and 34a-Het cultures showed enhanced osteoclast differentiation $(n=3)$. $\mathbf{i}$, Serum CTX-1 (2-month-old, male, $n=6) . \mathbf{j}-1, \mu C T$ of the tibiae (2-month-old, male, $\mathrm{n}=4$ ). j, Images. k, Trabecular bone parameters. l, Cortical BV/TV. m, Serum P1NP (2month-old, male, $n=6$ ). Error bars, SD. 


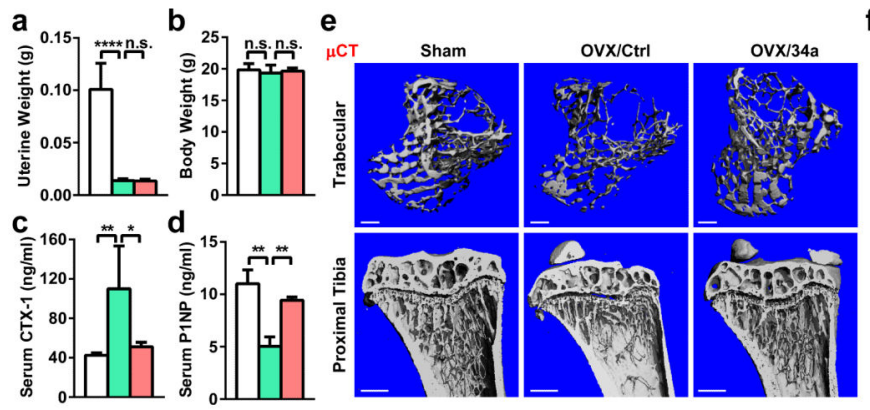

f 口 Sham $\square$ ovx/ctrl $\square$ ovx/34a

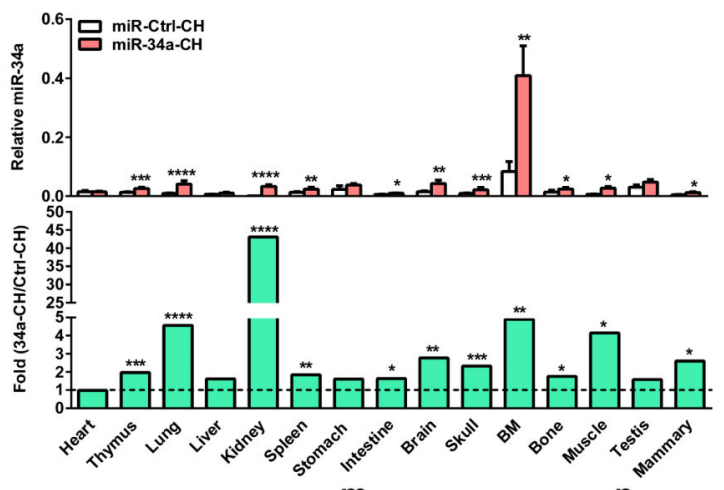

ロ Sham/Ctrl 口ovx/Ctrl $\square$ ovx/34a-Tie2-Tg

h

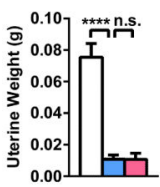

i $\overline{\bar{\varepsilon}}^{60} 7$ 然管

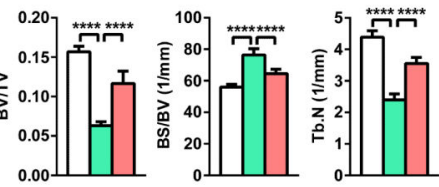

${ }^{0.04}$
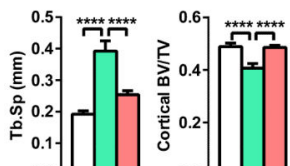

j
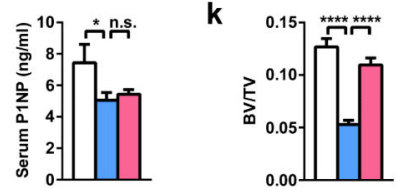

I $6.0 \times 10^{7} 7 \cdot \mathrm{Ct}$
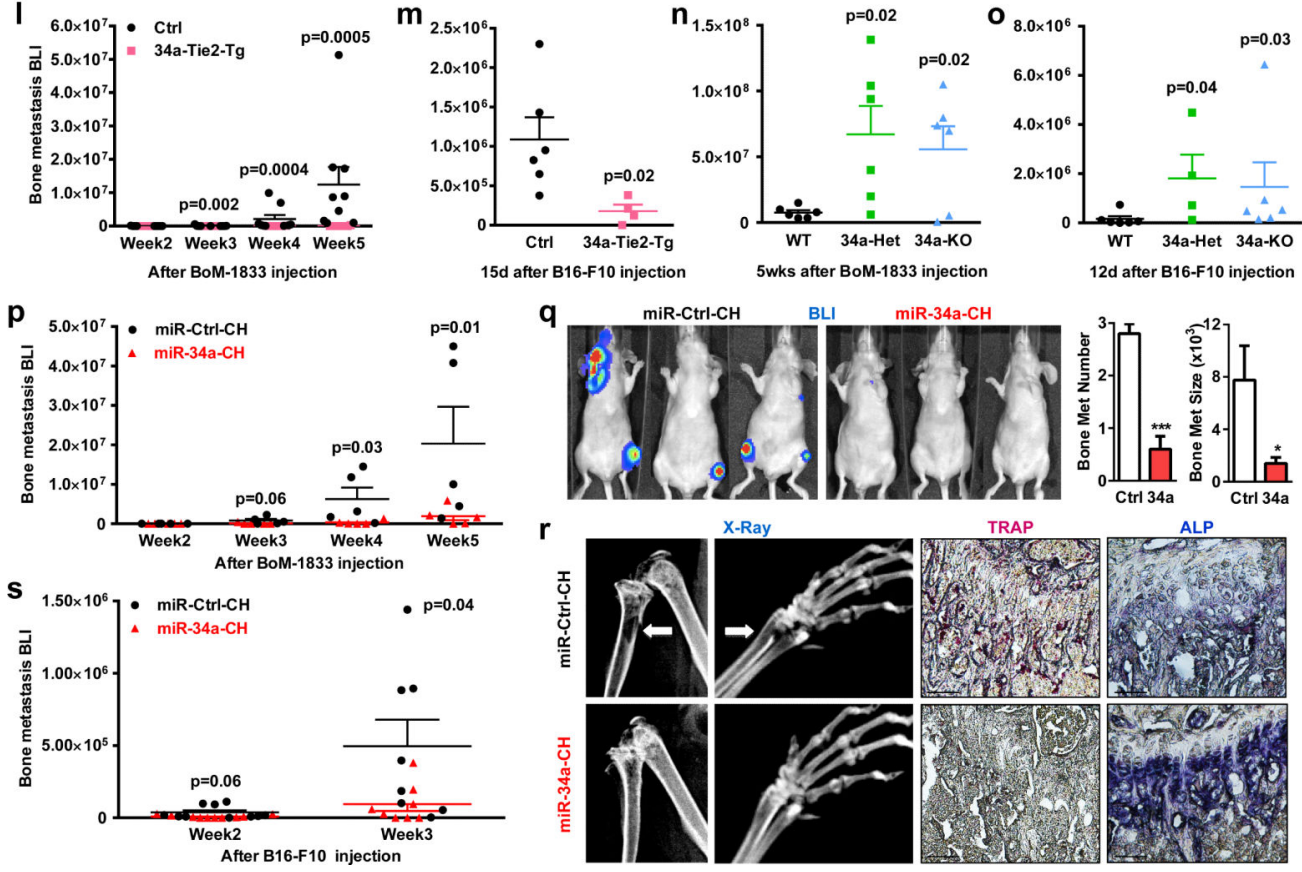

Figure 3. miR-34a Attenuates Osteoporosis and Cancer Bone Metastases

a-f, OVX or sham operation was performed on 10-week-old female mice. Three days post-

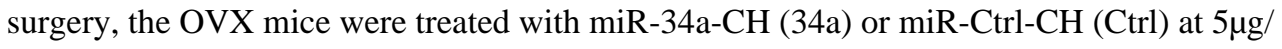
mouse twice/week for 5 weeks $(n=5)$. a, Uterine weight. b, Body weight. c, Serum CTX-1. d, Serum P1NP. e, $\mu$ CT images. f, Trabecular bone parameters. $\mathbf{g}$, miR-34a levels in each tissue from miR-34a-CH- vs. miR-Ctrl-CH-treated mice $72 \mathrm{hrs}$ after a single injection $(\mathrm{n}=3)$. Top, mature miR-34a levels; Bottom, fold induction. h-k, 34a-Tie2-Tg mice or controls (3month-old, female, $\mathrm{n}=7$ ) were subjected to OVX and analyzed 5 weeks post-surgery. $\mathbf{h}$, 
Uterine weight. i, Serum CTX-1. j, Serum P1NP. k, BV/TV by $\mu$ CT. l, Xenograft of MDA231-BoM-1833 cells into 34a-Tie2-Tg ( $n=8)$ or control $(n=9)$. m, Allograft of B16F10 cells into 34a-Tie2-Tg ( $n=4)$ or control $(n=6)$. $\mathbf{n}$, MDA231-BoM-1833 cells in 34a-KO $(n=6), 34 a-H e t(n=6)$ or control $(n=6) ., o, B 16-F 10$ in 34a-KO $(n=6), 34 a-H e t ~(n=4)$ or control $(n=6)$. p-r, Bone metastasis of MDA231-BoM-1833 cells was attenuated by

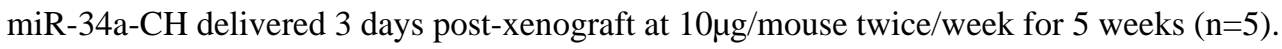
p, BLI signal. q, Left, BLI images; Right, bone metastases number and size. r, X-ray images and histology images for TRAP and ALP (alkaline phosphatase) staining. Arrows indicate osteolytic lesions. s, Bone metastasis of B16-F10 cells was attenuated by miR-34a-CH delivered at $5 \mu \mathrm{g} / \mathrm{mouse}$ twice/week for 4 weeks starting 1 week before cancer cell injection $(\mathrm{n}=8)$. l-s, Statistical analyses were performed with Mann Whitney Test and are shown as mean \pm SD with $\mathrm{p}$ value illustrated. a,c,d,f,h-k,l,n,o,p $\mathrm{p}<0.05$ by ANOVA. 

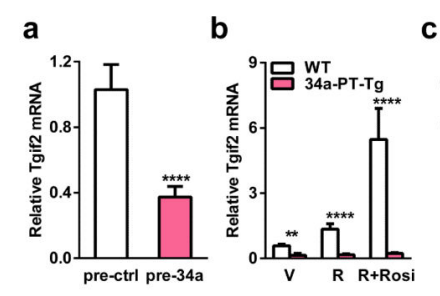
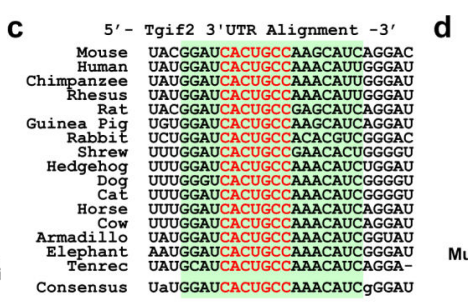

d
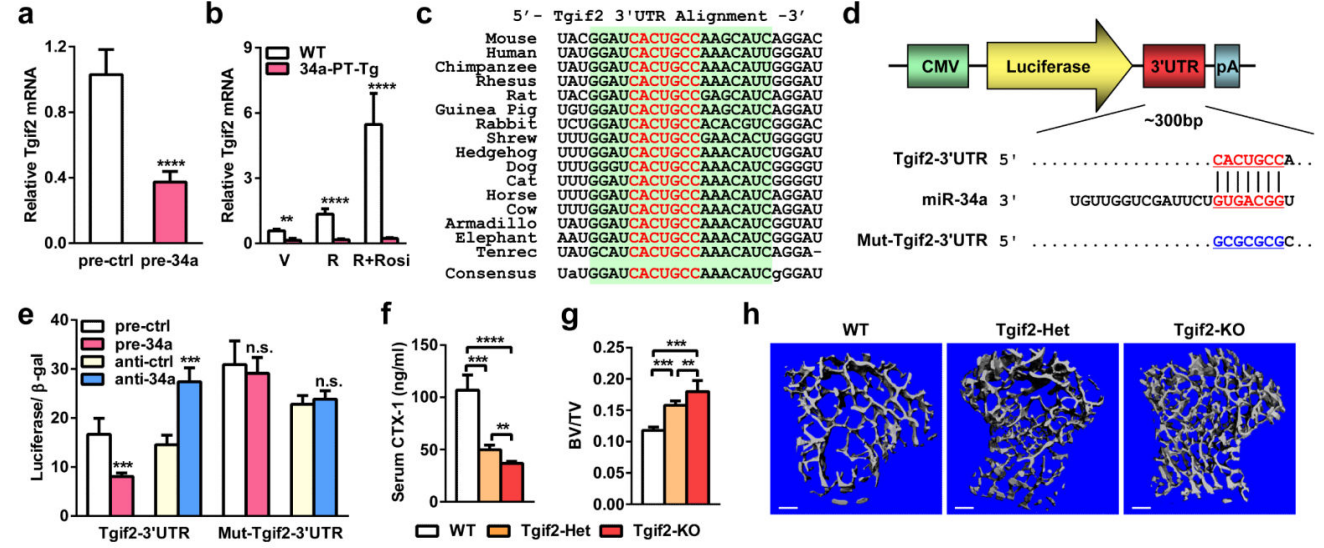

f

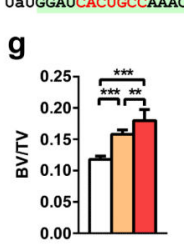

$\mathbf{h}$
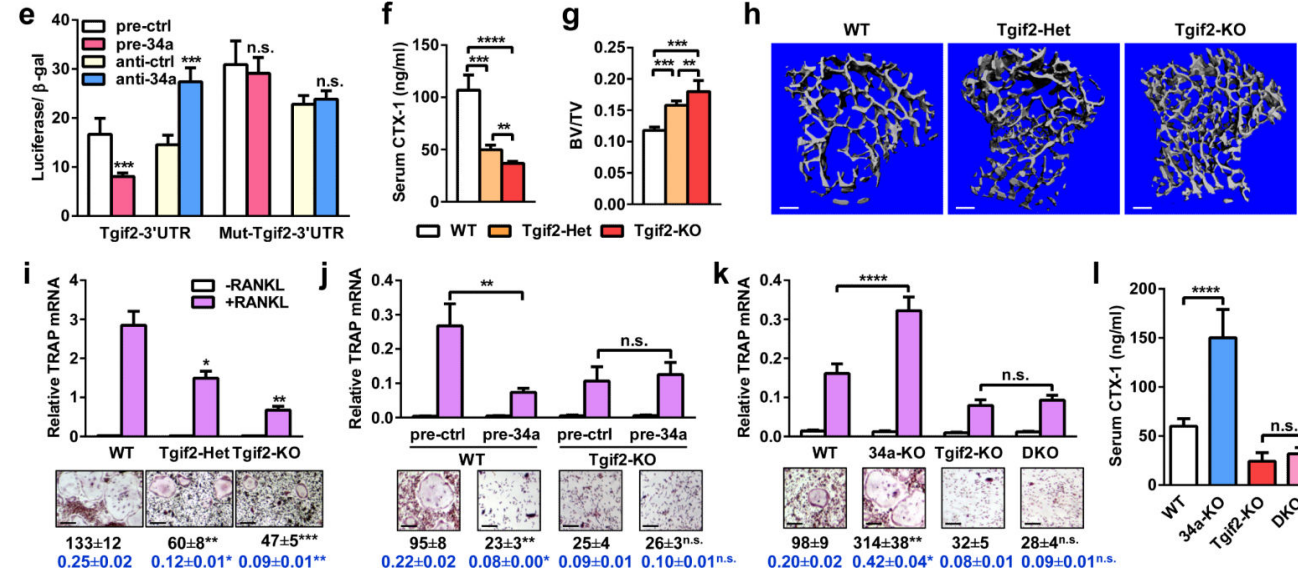

口 WT $\square$ Tgif2-Het $\square$ Tgif2-Ko
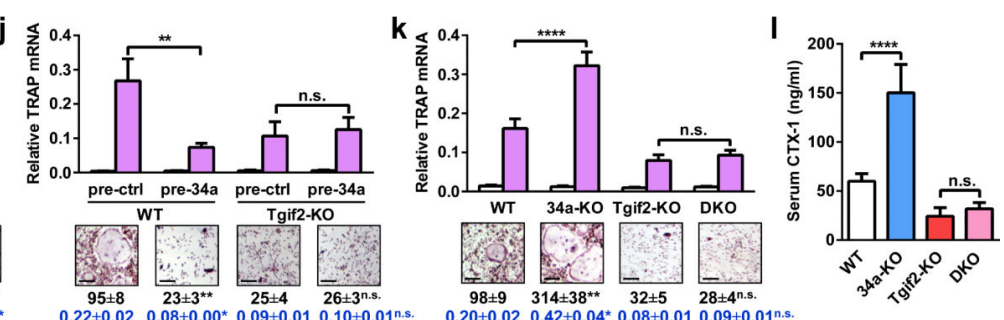

m

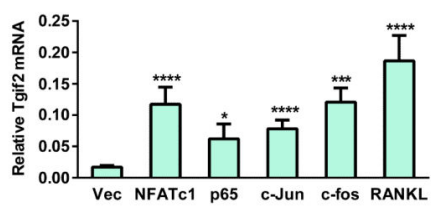

n $\left.\widetilde{0}^{15}\right\rceil$ 吕 +RANKLL

NFATC1-RE2

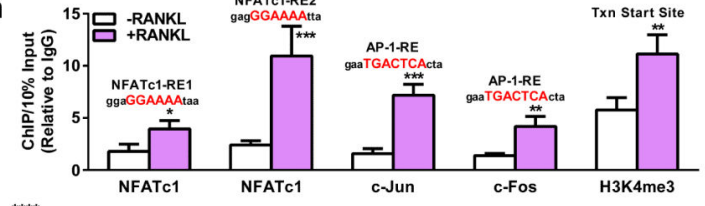

o
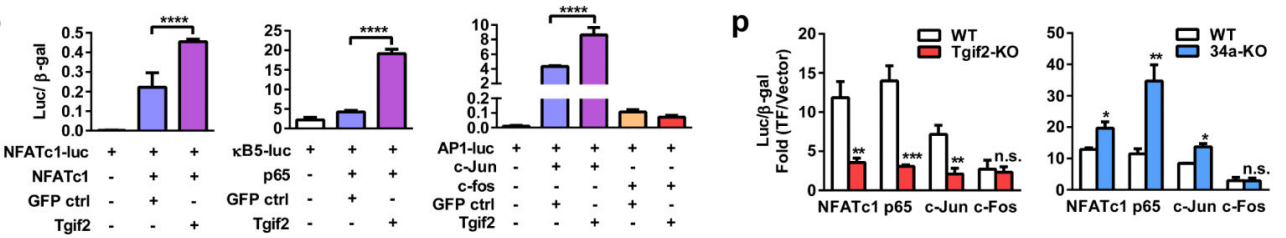

q

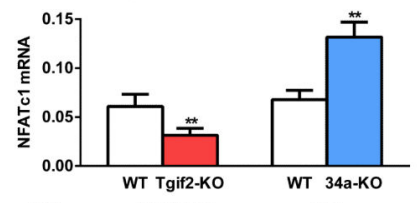

$\mathbf{r}$

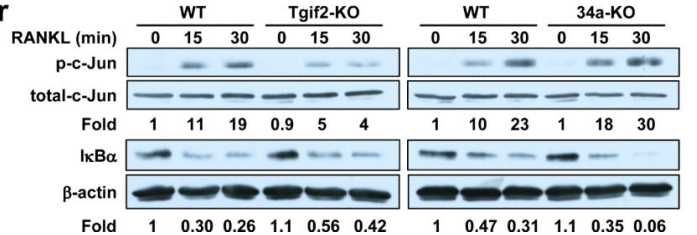

s

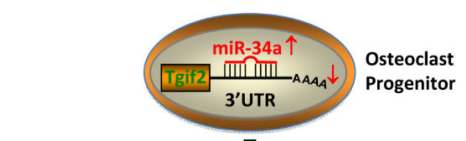

OSTEOPOROSIS $\downarrow$ BONE METASTASIS $\downarrow$

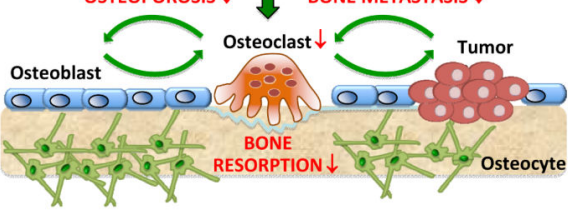

Figure 4. Tgif2 is an Essential miR-34a Direct Target and a Pro-Osteoclastogenic Factor a, Tgif2 expression was inhibited by pre-miR-34a in osteoclast cultures $(n=3)$. b, Tgif2 expression in WT and 34a-PT-Tg osteoclast cultures $(n=3)$. c, Sequence alignment of the Tgif2 3'UTR. d, A diagram of Tgif2 3'UTR reporters. e, Luciferase readout from WT or mutant Tgif2 3'UTR reporter co-transfected in HEK293 cells with pre-miR-34a or antimiR-34a (n=3). f-h, Comparison of Tgif2-KO, Tgif2-Het and WT control mice (1.5-monthold, male, n=7). f. Serum CTX-1. g-h, $\mu$ CT of tibiae. g, Trabecular BV/TV. h, Images of the

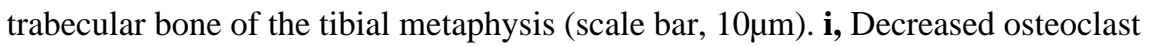


differentiation in Tgif2-KO and Tgif2-Het cultures $(n=3)$. j, Tgif2-KO cultures were resistant to the anti-osteoclastogenic effects of premiR-34a $(n=3)$. $\mathbf{i}-\mathbf{j}$, Top, TRAP expression; Bottom, TRAP staining, osteoclast number (black) and resorptive activity (blue). k-l, Tgif2/34a double knockout (DKO) mice were compared with WT, Tgif2-KO or 34a-KO (2-month-old, male, $n=4)$. k, Osteoclast differentiation. $\mathbf{l}$, Serum CTX-1. m, Tgif2 mRNA in RAW264.7 cells following transfection of transcription factors $(n=3) . \mathbf{n}$, ChIP of transcription factor binding and $\mathrm{H} 3 \mathrm{~K} 4 \mathrm{me} 3$ levels at the endogenous Tgif 2 promoter in RAW264.7 cells 3d after RANKL treatment $(n=6)$; txn, transcription. o, Transcription factor was co-transfected into 293 cells with its luciferase reporter, together with Tgif 2 or a GFP control $(n=6)$. p, Luciferase reporter was transfected into WT, Tgif2-KO or 34a-KO osteoclast cultures $(n=6)$. q-r, NFATc1 mRNA $(\mathbf{q}, n=3)$, c-Jun phosphorylation and I $\kappa$ Ba degradation (r) in WT, Tgif2-KO or 34a-KO osteoclast cultures. Ratios of p-c-Jun/total-c-

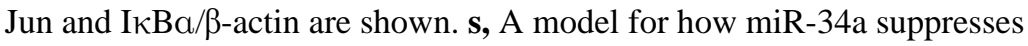
osteoclastogenesis. Error bars, SD. 
a

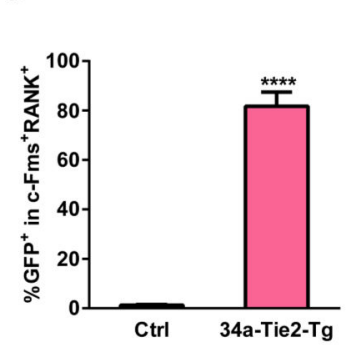

d

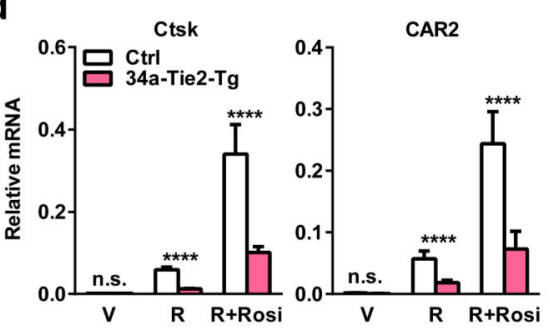

h

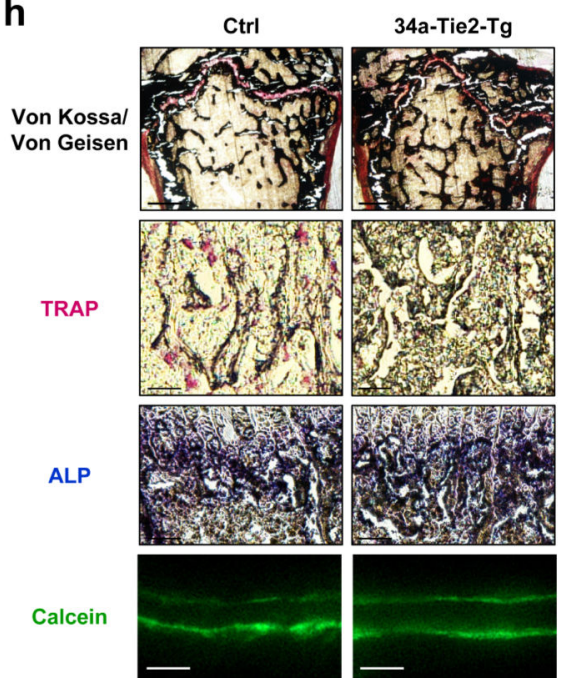

b

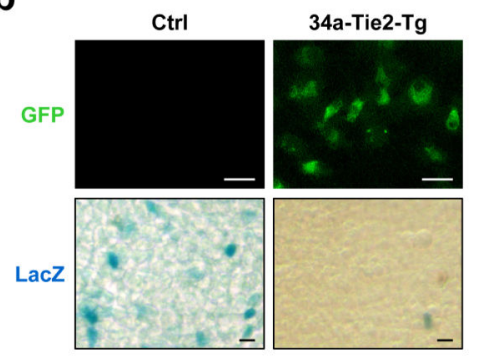

C

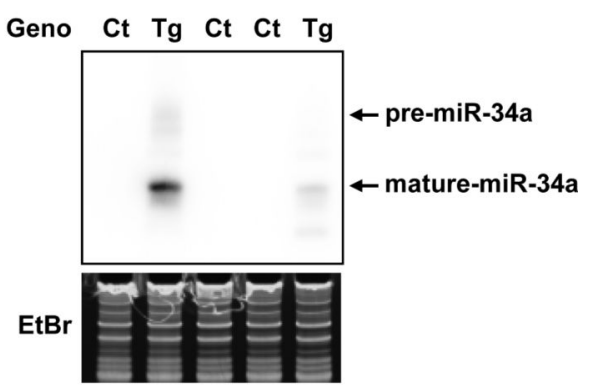

e

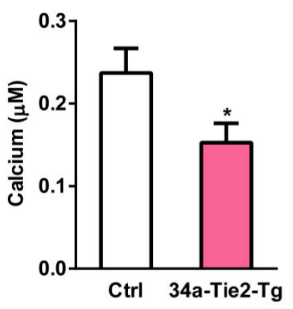

f

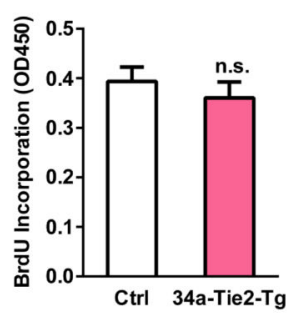

g

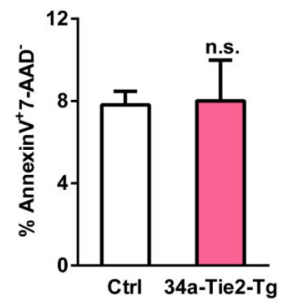

i

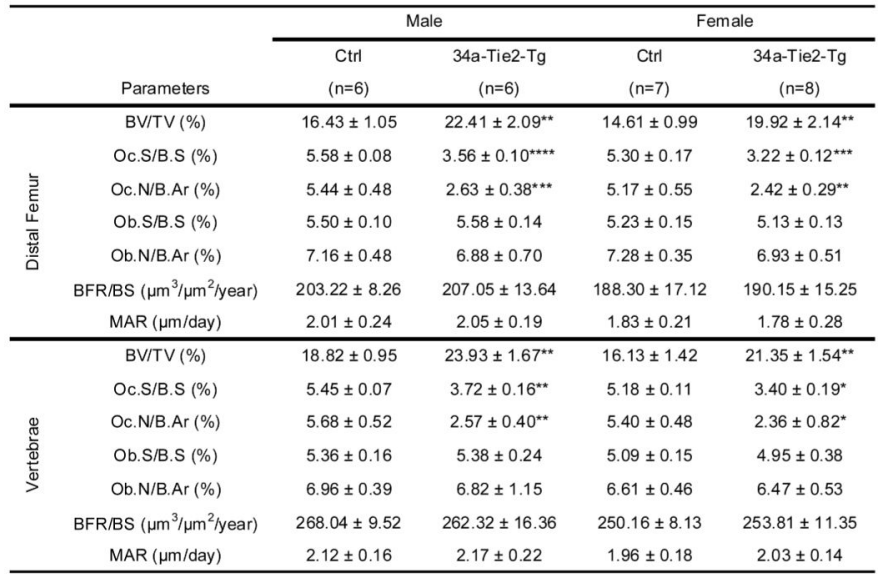

Extended Data Figure 1. Additional analyses of 34a-Tie2-Tg mice

a-c, Further characterization of the transgene expression in 34a-Tie2-Tg mice. a, FACS analysis of the percentage of $\mathrm{GFP}^{+}$bone marrow osteoclast progenitors (c-Fms ${ }^{+} \mathrm{RANK}^{+}$) in 34a-Tie2-Tg mice and "transgene only, no cre" control $(n=3)$. b, Images showing GFP and LacZ expression in osteoclast progenitors from 34a-Tie2-Tg mice $\left(\mathrm{GFP}^{+} \mathrm{LacZ}^{-}\right)$and

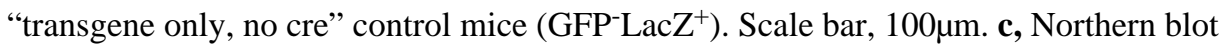
analysis confirmed miR-34a over-expression in the hematopoietic bone marrow cells of 34aTie2-Tg mice. Ct, control; Tg, 34a-Tie2-Tg; EtBr, ethidium bromide. d, QPCR analysis of mRNA expression of additional osteoclast marker genes $(n=3)$. e, Osteoclast function analysis. Bone marrow osteoclast differentiation was conducted in OsteoAssay bone plates (Lonza), and osteoclast activity was quantified as calcium release using CalciFluo ELISA assay (Lonza) $(n=8$, mean \pm s.e.). f, Osteoclast proliferation was not affected, quantified by BrdU incorporation ( $\mathrm{n}=6$ ). $\mathbf{g}$, Osteoclast apoptosis was not affected, quantified by FACS analysis of AnnexinV ${ }^{+} 7-\mathrm{AAD}^{-}$cells $(\mathrm{n}=6)$. $\mathbf{h}-\mathbf{i}$, Static and dynamic histomorphometry. $\mathbf{h}$, 
Representative images of distal femur sections ( 2 month old, male). Scale bars, $1 \mathrm{~mm}$ for Von Kossa images; $10 \mu \mathrm{m}$ for TRAP, ALP and Calcein images. i, Quantification of parameters at distal femur and vertebrae in 2-month-old male and female mice. 

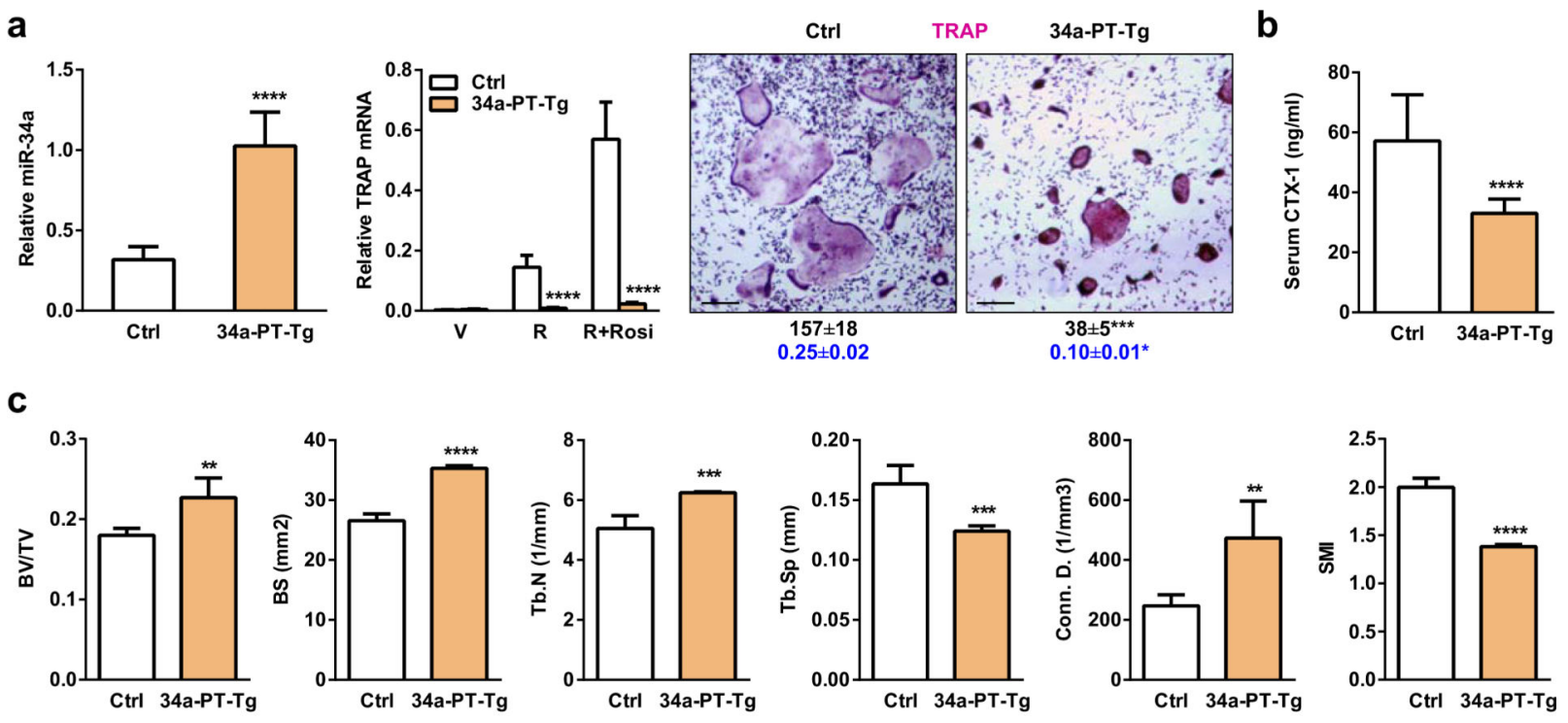

d

\begin{tabular}{|c|c|c|c|c|c|}
\hline & \multirow[b]{2}{*}{ Parameters } & \multicolumn{2}{|c|}{ Male } & \multicolumn{2}{|c|}{ Female } \\
\hline & & $\begin{array}{c}\text { Ctrl } \\
(n=10)\end{array}$ & $\begin{array}{c}\text { 34a-PT-Tg } \\
(n=10)\end{array}$ & $\begin{array}{c}\mathrm{Ctrl} \\
(\mathrm{n}=8)\end{array}$ & $\begin{array}{c}\text { 34a-PT-Tg } \\
(n=8)\end{array}$ \\
\hline \multirow{7}{*}{ 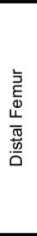 } & BV/TV (\%) & $17.29 \pm 0.68$ & $22.56 \pm 2.14^{\star \star}$ & $16.12 \pm 1.53$ & $20.44 \pm 1.95^{*}$ \\
\hline & Oc.S/B.S (\%) & $5.82 \pm 0.09$ & $3.47 \pm 0.16^{\star \star \star}$ & $5.31 \pm 0.14$ & $3.02 \pm 0.08^{n * *}$ \\
\hline & Oc.N/B.Ar (\%) & $6.03 \pm 0.52$ & $2.91 \pm 0.25^{* \star *}$ & $6.25 \pm 0.87$ & $2.88 \pm 0.32^{* *}$ \\
\hline & Ob.S/B.S (\%) & $6.22 \pm 0.18$ & $6.36 \pm 0.24$ & $6.06 \pm 0.35$ & $6.19 \pm 0.52$ \\
\hline & Ob.N/B.Ar (\%) & $7.41 \pm 0.56$ & $7.35 \pm 0.69$ & $7.22 \pm 0.86$ & $7.31 \pm 0.78$ \\
\hline & BFR/BS $\left(\mu \mathrm{m}^{3} / \mu \mathrm{m}^{2} /\right.$ year $)$ & $236.09 \pm 24.15$ & $244.27 \pm 17.96$ & $225.52 \pm 18.32$ & $232.06 \pm 21.72$ \\
\hline & $\operatorname{MAR}(\mu \mathrm{m} /$ day $)$ & $2.46 \pm 0.25$ & $2.53 \pm 0.28$ & $2.32 \pm 0.18$ & $2.40 \pm 0.25$ \\
\hline \multirow{7}{*}{ 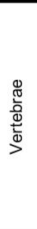 } & $\mathrm{BV} / \mathrm{TV}(\%)$ & $19.02 \pm 1.45$ & $23.48 \pm 1.06^{* *}$ & $18.26 \pm 1.26$ & $22.06 \pm 1.15^{\text {t* }}$ \\
\hline & Oc.S/B.S (\%) & $6.32 \pm 0.12$ & $3.67 \pm 0.23^{\star \star}$ & $5.99 \pm 0.25$ & $3.17 \pm 0.14^{* *}$ \\
\hline & Oc.N/B.Ar (\%) & $6.54 \pm 0.62$ & $3.05 \pm 0.31^{\star \star}$ & $6.76 \pm 0.35$ & $3.02 \pm 0.26^{\star \star}$ \\
\hline & Ob.S/B.S (\%) & $7.88 \pm 0.27$ & $7.79 \pm 0.26$ & $6.98 \pm 0.52$ & $6.94 \pm 0.38$ \\
\hline & Ob.N/B.Ar $(\%)$ & $8.69 \pm 0.62$ & $8.77 \pm 0.78$ & $8.06 \pm 0.72$ & $8.10 \pm 0.57$ \\
\hline & BFR/BS $\left(\mu \mathrm{m}^{3} / \mu \mathrm{m}^{2} /\right.$ year $)$ & $242.15 \pm 10.62$ & $247.32 \pm 16.47$ & $228.21 \pm 16.30$ & $230.14 \pm 21.06$ \\
\hline & $\operatorname{MAR}(\mu \mathrm{m} /$ day $)$ & $2.72 \pm 0.18$ & $2.78 \pm 0.21$ & $2.56 \pm 0.18$ & $2.59 \pm 0.17$ \\
\hline
\end{tabular}

Extended Data Figure 2. Effects of miR-34a over-expression using additional cre driver targeting osteoclast progenitors

34a-PT-Tg mice were generated using PPAR $\gamma$-tTA-TRE-cre driver. a, Bone marrow osteoclast differentiation assays. Left, mature miR-34a level $(n=3)$; middle, TRAP mRNA expression $(n=3)$; right, TRAP staining of differentiation cultures, quantification of mature osteoclast numbers per well in 24-well plates (black, $n=3$ ), and quantification of bone resorptive activity by calcium release from bone plate into culture medium $(\mu \mathrm{M})($ blue, $n=6)$. b, Serum CTX-1 bone resorption marker (2-month-old males, $\mathrm{n}=10$ ). $\mathbf{c}, \mu \mathrm{CT}$ analysis of the trabecular bone in proximal tibiae (2-month-old males, $n=4)$. d, Histomorphometry of the distal femur and vertebrae in 2-month-old mice. 
a

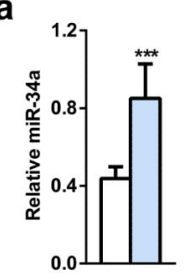

$\left.{ }^{0.4}\right\rceil$ 口 $34 \mathrm{a}$-trl

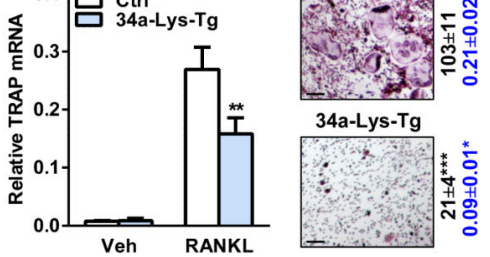

b

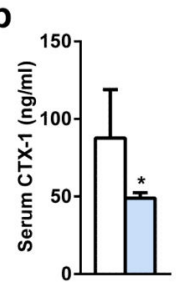

C

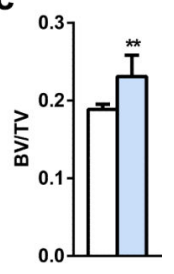

d

\begin{tabular}{|c|c|c|c|c|c|}
\hline & \multirow[b]{2}{*}{ Parameters } & \multicolumn{2}{|c|}{ Male } & \multicolumn{2}{|c|}{ Female } \\
\hline & & $\begin{array}{l}\mathrm{Ctrl} \\
(n=8)\end{array}$ & $\begin{array}{c}\text { 34a-Lys-Tg } \\
\quad(n=8)\end{array}$ & $\begin{array}{c}\mathrm{Ctrl} \\
(\mathrm{n}=6)\end{array}$ & $\begin{array}{c}\text { 34a-Lys-Tg } \\
\quad(n=6)\end{array}$ \\
\hline \multirow{7}{*}{ 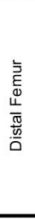 } & $\mathrm{BV} / \mathrm{TV}(\%)$ & $16.38 \pm 1.26$ & $21.67 \pm 2.85^{* *}$ & $14.22 \pm 0.86$ & $20.35 \pm 1.78^{* * *}$ \\
\hline & Oc.S/B.S (\%) & $5.35 \pm 0.13$ & $3.11 \pm 0.15^{* *}$ & $6.06 \pm 0.22$ & $3.91 \pm 0.39^{*}$ \\
\hline & Oc.N/B.Ar (\%) & $5.83 \pm 0.26$ & $3.36 \pm 0.22^{* *}$ & $6.25 \pm 0.38$ & $3.77 \pm 0.30^{\star \star}$ \\
\hline & Ob.S/B.S (\%) & $5.76 \pm 0.12$ & $5.66 \pm 0.16$ & $5.23 \pm 0.45$ & $5.26 \pm 0.39$ \\
\hline & Ob.N/B.Ar (\%) & $7.52 \pm 0.34$ & $7.65 \pm 0.53$ & $7.02 \pm 0.55$ & $7.14 \pm 0.46$ \\
\hline & BFR/BS $\left(\mu \mathrm{m}^{3} / \mu \mathrm{m}^{2} /\right.$ year $)$ & $229.27 \pm 9.62$ & $235.19 \pm 18.12$ & $213.37 \pm 11.35$ & $215.88 \pm 15.20$ \\
\hline & $\operatorname{MAR}(\mu \mathrm{m} /$ day) & $2.09 \pm 0.11$ & $2.14 \pm 0.26$ & $2.02 \pm 0.21$ & $2.07 \pm 0.18$ \\
\hline \multirow{7}{*}{ 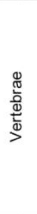 } & BV/TV (\%) & $18.62 \pm 1.02$ & $24.16 \pm 1.97^{* *}$ & $17.85 \pm 1.42$ & $22.92 \pm 1.86^{* *}$ \\
\hline & Oc.S/B.S (\%) & $5.08 \pm 0.48$ & $2.98 \pm 0.25^{*}$ & $5.66 \pm 0.38$ & $3.21 \pm 0.09^{* t *}$ \\
\hline & Oc.N/B.Ar (\%) & $5.27 \pm 0.35$ & $2.76 \pm 0.14^{* *}$ & $5.73 \pm 0.48$ & $2.68 \pm 0.12^{\star \star *}$ \\
\hline & Ob.S/B.S (\%) & $5.89 \pm 0.26$ & $5.75 \pm 0.38$ & $5.24 \pm 0.28$ & $5.27 \pm 0.33$ \\
\hline & Ob.N/B.Ar (\%) & $7.95 \pm 0.58$ & $7.83 \pm 0.62$ & $7.06 \pm 0.52$ & $7.13 \pm 0.47$ \\
\hline & BFR/BS $\left(\mu \mathrm{m}^{3} / / \mathrm{m}^{2} /\right.$ year $)$ & $236.55 \pm 15.28$ & $233.83 \pm 26.10$ & $216.75 \pm 13.02$ & $215.06 \pm 16.88$ \\
\hline & $\operatorname{MAR}(\mu \mathrm{m} / \mathrm{day})$ & $2.15 \pm 0.22$ & $2.17 \pm 0.16$ & $2.06 \pm 0.14$ & $2.08 \pm 0.19$ \\
\hline
\end{tabular}

e
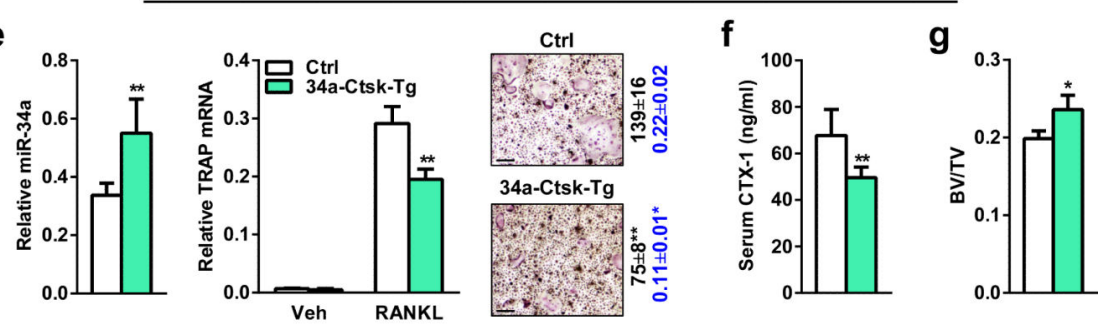

h

\begin{tabular}{|c|c|c|c|c|c|}
\hline & \multirow[b]{3}{*}{ Parameters } & \multicolumn{2}{|c|}{ Male } & \multicolumn{2}{|c|}{ Female } \\
\hline & & Ctrl & 34a-Ctsk- Tg & Ctrl & 34a-Ctsk-Tg \\
\hline & & $(n=8)$ & $(n=8)$ & $(n=7)$ & $(n=7)$ \\
\hline \multirow{7}{*}{ 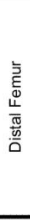 } & BV/TV (\%) & $14.52 \pm 1.08$ & $17.67 \pm 1.59^{*}$ & $12.96 \pm 0.73$ & $16.08 \pm 1.06^{\text {t* }}$ \\
\hline & Oc.S/B.S (\%) & $5.76 \pm 0.12$ & $4.15 \pm 0.20^{\star}$ & $5.88 \pm 0.25$ & $4.23 \pm 0.17^{*}$ \\
\hline & Oc.N/B.Ar (\%) & $4.93 \pm 0.43$ & $3.05 \pm 0.35^{\star}$ & $5.26 \pm 0.31$ & $3.37 \pm 0.16^{\star \star}$ \\
\hline & Ob.S/B.S (\%) & $5.84 \pm 0.15$ & $5.96 \pm 0.32$ & $5.26 \pm 0.31$ & $5.22 \pm 0.48$ \\
\hline & Ob.N/B.Ar (\%) & $8.22 \pm 0.86$ & $8.13 \pm 0.92$ & $7.98 \pm 0.54$ & $8.05 \pm 0.75$ \\
\hline & BFR/BS $\left(\mu \mathrm{m}^{3} / \mathrm{mm}^{2} /\right.$ year $)$ & $216.53 \pm 12.08$ & $219.01 \pm 9.43$ & $208.40 \pm 8.29$ & $209.26 \pm 14.80$ \\
\hline & $\operatorname{MAR}(\mu \mathrm{m} /$ day $)$ & $2.24 \pm 0.16$ & $2.30 \pm 0.24$ & $2.14 \pm 0.09$ & $2.16 \pm 0.20$ \\
\hline \multirow{7}{*}{ 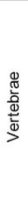 } & BV/TV (\%) & $16.35 \pm 0.96$ & $19.22 \pm 1.24^{*}$ & $15.08 \pm 0.73$ & $18.22 \pm 1.36^{*}$ \\
\hline & Oc.S/B.S (\%) & $5.25 \pm 0.38$ & $3.88 \pm 0.17^{\star}$ & $5.84 \pm 0.31$ & $4.33 \pm 0.26^{*}$ \\
\hline & Oc.N/B.Ar (\%) & $4.69 \pm 0.31$ & $2.72 \pm 0.16^{\star}$ & $5.06 \pm 0.20$ & $3.03 \pm 0.27^{*}$ \\
\hline & Ob.S/B.S (\%) & $6.23 \pm 0.38$ & $6.18 \pm 0.54$ & $5.96 \pm 0.47$ & $5.89 \pm 0.52$ \\
\hline & Ob.N/B.Ar (\%) & $9.53 \pm 0.61$ & $9.68 \pm 1.07$ & $8.67 \pm 0.52$ & $8.72 \pm 0.88$ \\
\hline & BFR/BS $\left(\mu \mathrm{m}^{3} / \mathrm{mm}^{2} /\right.$ year $)$ & $230.62 \pm 16.73$ & $229.65 \pm 18.63$ & $225.39 \pm 17.66$ & $228.75 \pm 18.02$ \\
\hline & $\operatorname{MAR}(\mu \mathrm{m} /$ day $)$ & $2.58 \pm 0.20$ & $2.62 \pm 0.18$ & $2.35 \pm 0.12$ & $2.32 \pm 0.15$ \\
\hline
\end{tabular}

Extended Data Figure 3. Effects of miR-34a over-expression using additional osteoclastic cre drivers

a-d, 34a-Lys-Tg mice were generated using Lysozyme-cre driver. e-h, 34a-Ctsk-Tg mice were generated using Ctsk-cre driver. a,e, Bone marrow osteoclast differentiation assays. Left, mature miR-34a level $(n=3)$; middle, TRAP mRNA expression $(n=3)$; right, TRAP staining of differentiation cultures, quantification of mature osteoclast numbers per well in 24-well plates (black, $n=3$ ), and quantification of bone resorptive activity by calcium release from bone plate into culture medium $(\mu \mathrm{M})$ (blue, $\mathrm{n}=6)$. b,f, Serum CTX-1 (2-month-old 
males; $\mathbf{b}, \mathrm{n}=5, \mathbf{f}, \mathrm{n}=8$ ). $\mathbf{c}, \mathbf{g}$, Trabecular BV/TV of proximal tibiae by $\mu \mathrm{CT}$ (2-month-old males; $\mathbf{c}, \mathrm{n}=4 ; \mathbf{g}, \mathbf{n = 4}$ ). $\mathbf{d}, \mathbf{h}$, Histomorphometry of the distal femur and vertebrae in 2-monthold mice. 
a

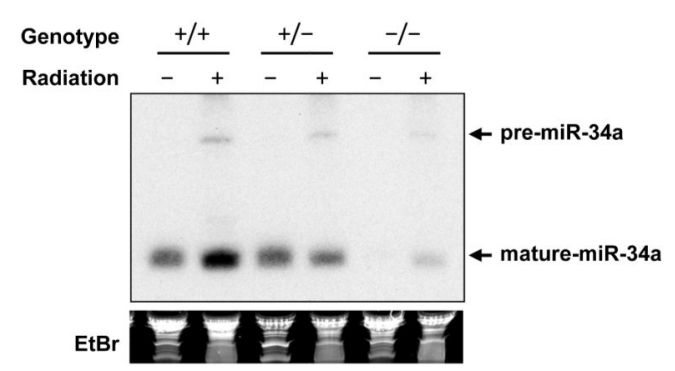

C

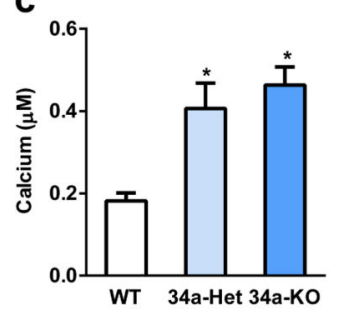

e

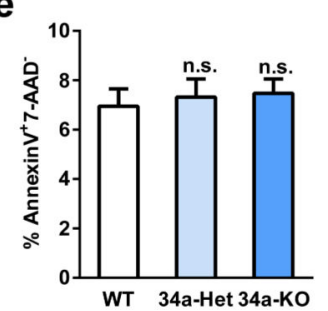

d

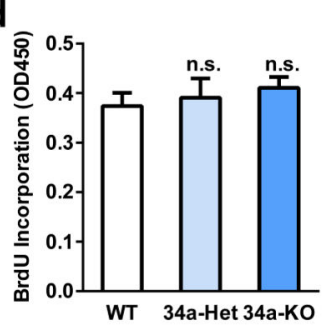

f

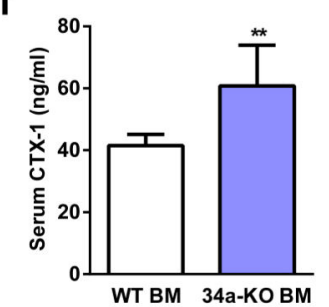

b
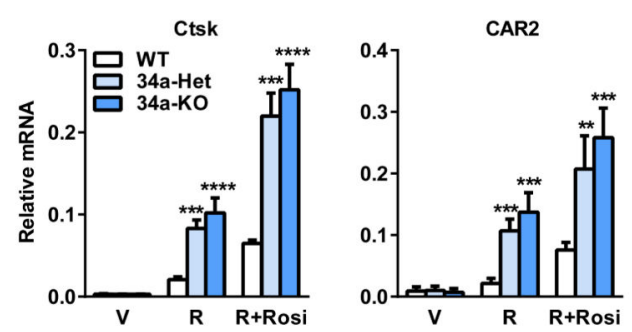

g

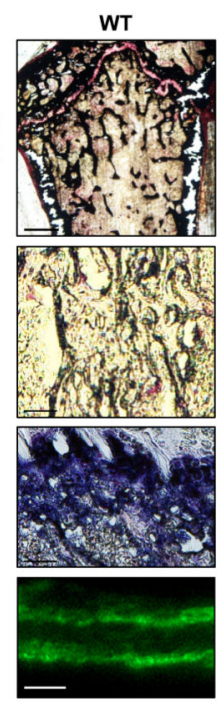

34a-Het

34a-KO
Von Kossal Von Geisen

TRAP
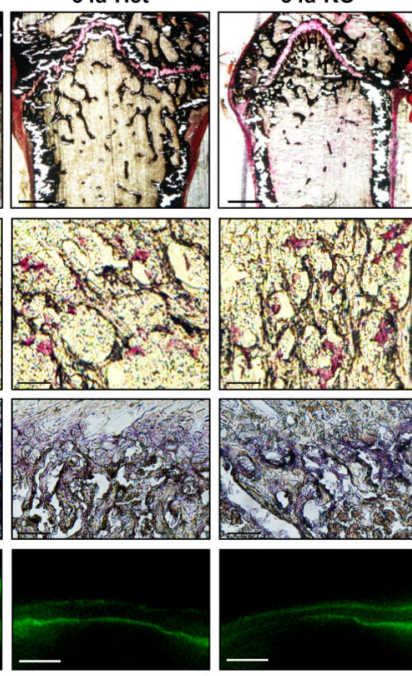

ALP

Calcein

h

\begin{tabular}{|c|c|c|c|c|c|c|c|}
\hline & \multirow[b]{3}{*}{ Parameters } & \multicolumn{3}{|c|}{ Male } & \multicolumn{3}{|c|}{ Female } \\
\hline & & WT & 34a-Het & $34 a-K O$ & WT & 34a-Het & $34 a-K O$ \\
\hline & & $(n=6)$ & $(n=6)$ & $(n=6)$ & $(n=7)$ & $(n=8)$ & $(n=7)$ \\
\hline \multirow{7}{*}{ 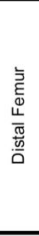 } & BV/TV (\%) & $15.96 \pm 1.73$ & $10.09 \pm 1.64^{* \star}$ & $8.82 \pm 1.49^{\star \star \star}$ & $13.18 \pm 1.96$ & $7.88 \pm 1.32^{\star \star}$ & $6.45 \pm 1.83^{\star \star}$ \\
\hline & Oc.S/B.S (\%) & $5.92 \pm 0.10$ & $8.61 \pm 0.34^{\star \star \star \star}$ & $9.21 \pm 0.47^{\star \star \star \star * *}$ & $5.84 \pm 0.16$ & $8.57 \pm 0.49^{\star \star \star \star}$ & $9.56 \pm 0.60^{\star \star \star *}$ \\
\hline & Oc.N/B.Ar (\%) & $4.56 \pm 0.47$ & $7.00 \pm 0.32^{* *}$ & $7.40 \pm 0.52^{\star \star \star *}$ & $4.38 \pm 0.38$ & $6.92 \pm 0.25^{\star \star}$ & $7.83 \pm 0.31^{\star \star * *}$ \\
\hline & Ob.S/B.S (\%) & $5.59 \pm 0.04$ & $4.43 \pm 0.30^{\star \star \star}$ & $4.14 \pm 0.30^{\star \star \star \star}$ & $5.63 \pm 0.25$ & $4.48 \pm 0.46^{\star \star}$ & $4.20 \pm 0.18^{\star \star \star}$ \\
\hline & Ob.N/B.Ar (\%) & $11.85 \pm 0.86$ & $7.99 \pm 0.23^{* \star \star \star \star ~}$ & $6.75 \pm 0.56^{\star \star * * *}$ & $9.31 \pm 0.55$ & $6.87 \pm 0.69^{* \star}$ & $6.21 \pm 0.43^{\star \star \star *}$ \\
\hline & BFR/BS $\left(\mu \mathrm{m}^{3} / \mu \mathrm{m}^{2} /\right.$ year $)$ & $197.43 \pm 8.33$ & $148.95 \pm 5.17^{\star \star * *}$ & $135.06 \pm 4.31^{* * *}$ & $186.14 \pm 9.06$ & $141.20 \pm 6.21^{* * *}$ & $123.37 \pm 7.60^{* * *}$ \\
\hline & $\operatorname{MAR}(\mu \mathrm{m} /$ day $)$ & $2.02 \pm 0.15$ & $1.62 \pm 0.09^{* \star}$ & $1.55 \pm 0.11^{\star \star}$ & $1.91 \pm 0.12$ & $1.54 \pm 0.23^{*}$ & $1.32 \pm 0.17^{\star \star}$ \\
\hline \multirow{7}{*}{ 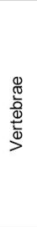 } & BV/TV (\%) & $18.21 \pm 2.01$ & $11.79 \pm 1.02^{* \star}$ & $9.12 \pm 0.38^{\star \star \star *}$ & $15.34 \pm 1.22$ & $8.54 \pm 0.60^{\star \star}$ & $7.18 \pm 0.68^{\text {t* }}$ \\
\hline & Oc.S/B.S (\%) & $5.56 \pm 0.22$ & $8.77 \pm 0.43^{* * *}$ & $9.38 \pm 0.33^{* \star *}$ & $5.39 \pm 0.14$ & $7.98 \pm 0.36^{\text {** }}$ & $8.67 \pm 0.28^{* * * *}$ \\
\hline & Oc.N/B.Ar (\%) & $4.35 \pm 0.26$ & $7.12 \pm 1.06^{*}$ & $7.56 \pm 0.38^{\star \star *}$ & $3.89 \pm 0.26$ & $6.77 \pm 0.43^{\star \star *}$ & $7.18 \pm 0.24^{\star \star \star *}$ \\
\hline & Ob.S/B.S (\%) & $5.23 \pm 0.32$ & $4.05 \pm 0.25^{*}$ & $3.86 \pm 0.29^{\star}$ & $5.44 \pm 0.18$ & $4.31 \pm 0.36^{*}$ & $4.05 \pm 0.14^{\star \star}$ \\
\hline & Ob.N/B.Ar (\%) & $9.71 \pm 0.97$ & $6.28 \pm 0.71^{*}$ & $5.84 \pm 0.68^{* *}$ & $8.77 \pm 0.40$ & $6.26 \pm 0.76^{\text {** }}$ & $5.75 \pm 0.88^{* *}$ \\
\hline & BFR/BS $\left(\mu \mathrm{m}^{3} / \mu \mathrm{m}^{2} /\right.$ year $)$ & $264.52 \pm 9.06$ & $214.18 \pm 4.35^{\star \star}$ & $202.61 \pm 7.28^{\star \star}$ & $258.79 \pm 5.74$ & $215.34 \pm 8.06^{\star \star}$ & $201.28 \pm 4.27^{\star \star \star}$ \\
\hline & $\operatorname{MAR}(\mu \mathrm{m} / \mathrm{day})$ & $2.31 \pm 0.18$ & $1.94 \pm 0.15^{*}$ & $1.82 \pm 0.08^{\star * *}$ & $2.10 \pm 0.21$ & $1.76 \pm 0.12^{\star \star *}$ & $1.64 \pm 0.10^{* *}$ \\
\hline
\end{tabular}

Extended Data Figure 4. Additional analyses of gene-trap miR-34a knockout mice a, Northern blot analysis confirmed decreased miR-34a expression in the miR-34a gene trap KO mice. Six-week-old female mice with corresponding genotypes were irradiated with a dose of $6 \mathrm{~Gy}$, and $4 \mathrm{~h}$ later the spleen was collected for RNA extraction. Northern blotting for miR-34a was performed as described (Chang TC et al. 2008, Nature Genetics 40:43-50). b, QPCR analysis of mRNA expression of additional osteoclast marker genes $(n=3)$. c, Osteoclast function analysis. Bone marrow osteoclast differentiation was conducted in OsteoAssay bone plates (Lonza), and osteoclast activity was quantified as calcium release using CalciFluo ELISA assay (Lonza) $(n=8$, mean \pm s.e.). d, Osteoclast proliferation was not 
affected, quantified by BrdU incorporation ( $n=6)$. e, Osteoclast apoptosis was not affected, quantified by FACS analysis of Annexin ${ }^{+} 7-\mathrm{AAD}^{-}$cells $(\mathrm{n}=6)$. f. WT mice transplanted with $34 \mathrm{a}-\mathrm{KO}$ bone marrow cells exhibited higher serum CTX-1 levels compared to WT mice transplanted with WT bone marrow cells ( $n=5$ recipients per group). $\mathbf{g}-\mathbf{h}$, Static and dynamic histomorphometry. g, Representative images of distal femur sections (2 month old, male). Scale bars, $1 \mathrm{~mm}$ for Von Kossa images; $10 \mu \mathrm{m}$ for TRAP, ALP and Calcein images. $\mathbf{h}$, Quantification of parameters at distal femur and vertebrae in 2-month-old male and female mice. 

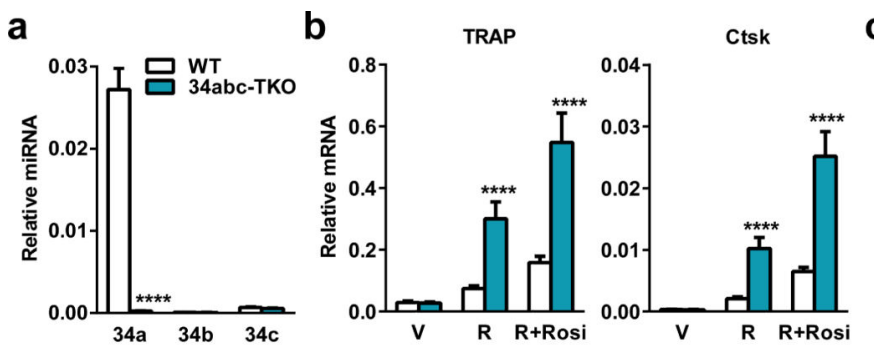

C
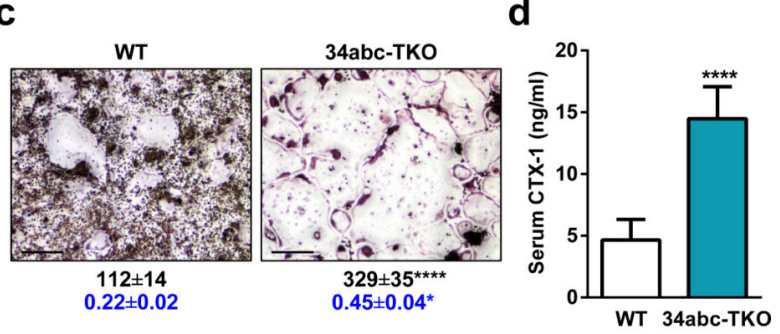
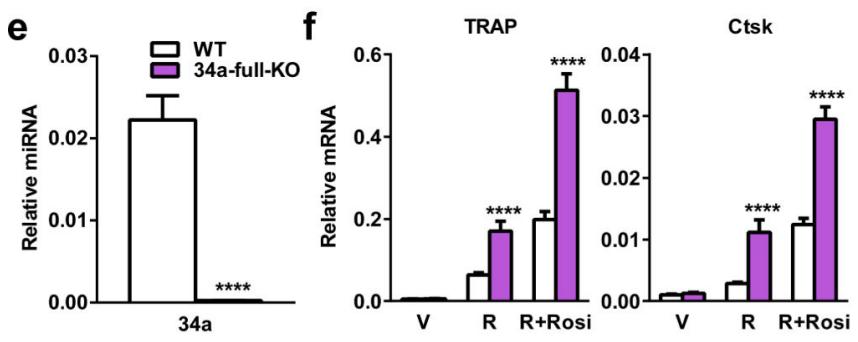

g
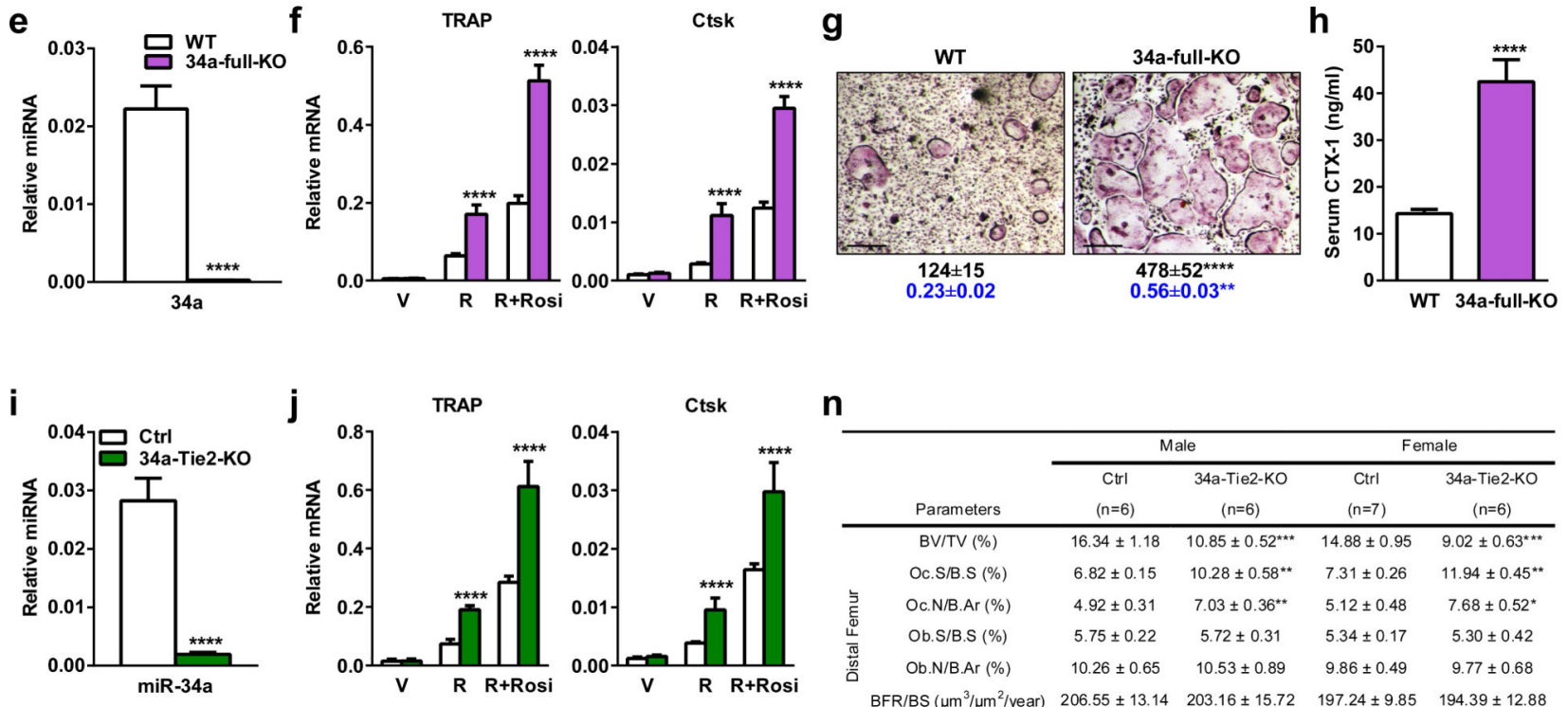

$\mathbf{n}$
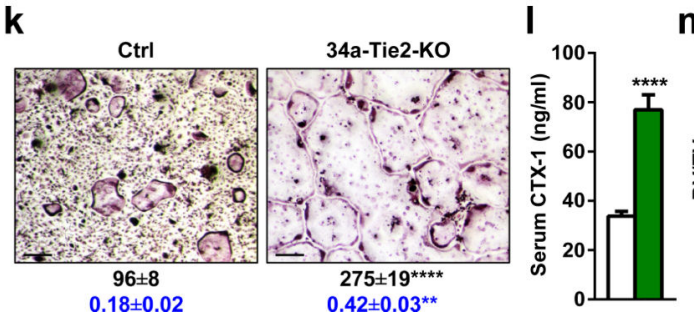

m

Extended Data Figure 5. Effects of targeted miR-34a deletion

a-d, Targeted miR-34a/b/c triple KO (34abc-TKO) mice were compared with WT control mice ( 5 month males, $n=4$ ). a-c, Bone marrow osteoclast differentiation assay. a, Expression of miR-34a was diminished while expression of miR-34b and miR-34c remained absent/low in osteoclast precursors on d3. b, Expression of osteoclast markers were increased. c, Number, size and resorptive activity of mature osteoclasts were increased. d, Serum CTX-1 was increased. e-h, Targeted full miR-34a KO (34a-full-KO) mice were compared with WT control mice ( 2 month females, $n=3$ ). e-g, Bone marrow osteoclast differentiation assay. e, Expression of miR-34a was diminished in osteoclast precursors on d3. f, Expression of osteoclast markers were increased. $\mathbf{g}$, Number, size and resorptive activity of mature osteoclasts were increased. h, Serum CTX-1 was increased. i-n, Conditional miR-34a KO mice by Tie2-cre (34a-Tie2-KO) were compared with littermate miR-34af/f control mice ( 2 month males, $n=6$ ). $\mathbf{i}-\mathbf{k}$, Bone marrow osteoclast differentiation assay. $\mathbf{i}$, miR-34a expression was reduced in osteoclast precursors on $\mathrm{d} 3$. $\mathbf{j}$, Expression of osteoclast markers were 
increased. k, Number, size and resorptive activity of mature osteoclasts were increased. l, Serum CTX-1 was increased. $\mathbf{m}$, Trabecular BV/TV of proximal tibiae by $\mu \mathrm{CT}$. $\mathbf{n}$, Histomorphometry of the distal femur and vertebrae. For $\mathbf{c}, \mathbf{g}, \mathbf{k}$, Mature osteoclasts were identified as multinucleated ( $>3$ nuclei) $\mathrm{TRAP}^{+}$(purple) cells. Scale bar, $25 \mu \mathrm{m}$. Quantification of osteoclast number/well is shown in black. Quantification of osteoclast resorptive activity by calcium release from bone to culture medium $(\mu \mathrm{M})$ is shown in blue. 
a

\begin{tabular}{|c|c|c|c|c|}
\hline & & Sham & OVX/Ctrl & $O V \times / 34 a$ \\
\hline & Parameters & $(n=5)$ & $(n=5)$ & $(n=5)$ \\
\hline \multirow{7}{*}{ 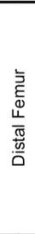 } & BV/TV (\%) & $13.21 \pm 0.68$ & $5.52 \pm 0.21^{\text {tht }}$ & $12.26 \pm 0.46^{++}$ \\
\hline & Oc.S/B.S (\%) & $5.63 \pm 0.13$ & $13.48 \pm 0.51^{t * \pm}$ & $6.22 \pm 0.14^{4+3+}$ \\
\hline & Oc.N/B.Ar $(\%)$ & $5.72 \pm 0.27$ & $12.42 \pm 0.69^{* * * x}$ & $6.65 \pm 0.42^{++}$ \\
\hline & Ob.S/B.S (\%) & $5.56 \pm 0.35$ & $3.27 \pm 0.12^{* * *}$ & $5.12 \pm 0.28^{+}$ \\
\hline & Ob.N/B.Ar (\%) & $6.16 \pm 0.75$ & $3.83 \pm 0.27^{*}$ & $5.53 \pm 0.32^{+}$ \\
\hline & BFR/BS $\left(\mathrm{um}^{3} / \mu \mathrm{m}^{2} /\right.$ year $)$ & $212.17 \pm 13.89$ & $125.06 \pm 9.65^{* *}$ & $189.90 \pm 8.31^{++}$ \\
\hline & $\operatorname{MAR}(\mu \mathrm{m} /$ day $)$ & $2.03 \pm 0.18$ & $1.45 \pm 0.06^{*}$ & $1.82 \pm 0.09^{+}$ \\
\hline \multirow{7}{*}{ 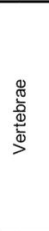 } & BV/TV (\%) & $15.56 \pm 0.72$ & $7.88 \pm 0.60^{* *}$ & $14.93 \pm 0.29^{++}$ \\
\hline & Oc.S/B.S (\%) & $5.24 \pm 0.31$ & $12.85 \pm 0.86^{* *}$ & $5.70 \pm 0.25^{++}$ \\
\hline & Oc.N/B.Ar $(\%)$ & $5.37 \pm 0.49$ & $12.25 \pm 0.86^{* * *}$ & $5.98 \pm 0.38^{+*}$ \\
\hline & Ob.S/B.S (\%) & $6.23 \pm 0.19$ & $3.98 \pm 0.38^{* *}$ & $5.81 \pm 0.23^{+}$ \\
\hline & Ob.N/B.Ar (\%) & $6.97 \pm 0.52$ & $4.29 \pm 0.33^{*}$ & $6.31 \pm 0.40^{+}$ \\
\hline & BFR/BS $\left(\mu \mathrm{m}^{3} / \mathrm{mm}^{2} /\right.$ year $)$ & $228.63 \pm 9.37$ & $134.68 \pm 11.80^{\text {** }}$ & $195.26 \pm 10.34^{+}$ \\
\hline & $\operatorname{MAR}(\mu \mathrm{m} / \mathrm{day})$ & $2.45 \pm 0.15$ & $1.57 \pm 0.12^{\star}$ & $2.19 \pm 0.16^{+}$ \\
\hline
\end{tabular}

b

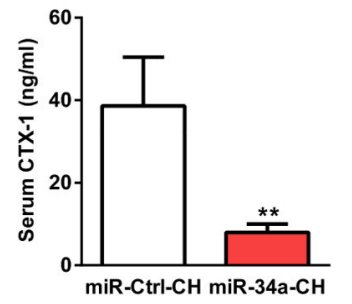

C

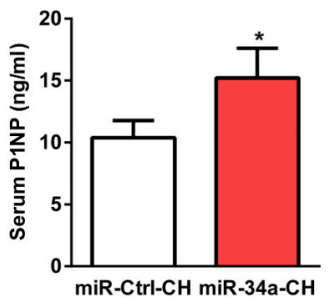

d

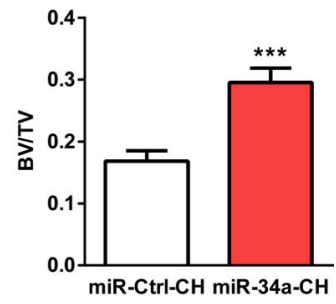

e

\begin{tabular}{|c|c|c|c|c|}
\hline & Parameters & $\begin{array}{c}\text { Sham/Ctrl } \\
(n=7)\end{array}$ & $\begin{array}{l}\text { OVX/Ctrl } \\
(n=7)\end{array}$ & $\begin{array}{c}\mathrm{OVX} / 34 \mathrm{a}-\mathrm{Tie} 2-\mathrm{Tg} \\
(\mathrm{n}=7)\end{array}$ \\
\hline \multirow{7}{*}{ 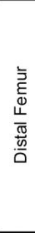 } & BV/TV (\%) & $11.52 \pm 0.49$ & $4.06 \pm 0.18^{* * * *}$ & $10.91 \pm 0.37^{+++}$ \\
\hline & Oc.S/B.S (\%) & $6.27 \pm 0.09$ & $15.66 \pm 0.28^{\star \star \star \star \star}$ & $6.15 \pm 0.11^{+++}$ \\
\hline & Oc.N/B.Ar (\%) & $6.33 \pm 0.28$ & $13.52 \pm 0.61^{\star \star \star \star}$ & $6.42 \pm 0.35^{++}$ \\
\hline & Ob.S/B.S (\%) & $5.02 \pm 0.26$ & $3.14 \pm 0.18^{* *}$ & $3.37 \pm 0.33$ \\
\hline & Ob.N/B.Ar (\%) & $5.88 \pm 0.68$ & $3.36 \pm 0.42^{\star}$ & $3.52 \pm 0.38$ \\
\hline & BFR/BS $\left(\mu \mathrm{m}^{3} / \mu \mathrm{m}^{2} /\right.$ year $)$ & $198.06 \pm 11.56$ & $119.77 \pm 6.15^{\star \star}$ & $126.81 \pm 9.40$ \\
\hline & $\operatorname{MAR}(\mu \mathrm{m} / \mathrm{day})$ & $2.17 \pm 0.15$ & $1.40 \pm 0.07^{\star}$ & $1.46 \pm 0.12$ \\
\hline \multirow{7}{*}{$\begin{array}{l}\frac{0}{\pi} \\
\frac{\pi}{0} \\
\frac{\pi}{2} \\
>\end{array}$} & $\mathrm{BV} / \mathrm{TV}(\%)$ & $13.66 \pm 0.57$ & $5.71 \pm 0.32^{\star \star \star}$ & $12.78 \pm 0.63^{++}$ \\
\hline & Oc.S/B.S (\%) & $6.05 \pm 0.21$ & $15.27 \pm 0.59^{\star \star \star \star}$ & $6.34 \pm 0.47^{++}$ \\
\hline & Oc.N/B.Ar $(\%)$ & $6.06 \pm 0.32$ & $12.85 \pm 0.89^{*+* *}$ & $5.95 \pm 0.28^{++}$ \\
\hline & Ob.S/B.S (\%) & $5.94 \pm 0.18$ & $3.77 \pm 0.09^{* *}$ & $3.90 \pm 0.17$ \\
\hline & Ob.N/B.Ar (\%) & $6.57 \pm 0.35$ & $4.26 \pm 0.24^{\star}$ & $4.31 \pm 0.19$ \\
\hline & BFR/BS $\left(\mu \mathrm{m}^{3} / \mu \mathrm{m}^{2} /\right.$ year $)$ & $203.55 \pm 9.67$ & $132.08 \pm 10.25^{\star \star *}$ & $137.20 \pm 11.22$ \\
\hline & $\operatorname{MAR}(\mu \mathrm{m} /$ day $)$ & $2.52 \pm 0.18$ & $1.78 \pm 0.12^{*}$ & $1.75 \pm 0.29$ \\
\hline
\end{tabular}

* compares OVX/Ctrl with Sham/Ctrl; ${ }^{+}$compares OVX/34a-Tie2-Tg with OVX/Ctrl

Extended Data Figure 6. Anti-osteoporosis effects of miR-34a

a, Histomorphometry of the distal femur and vertebrae in OVX mice treated with miR-34a$\mathrm{CH}$ nanoparticles. OVX or sham operation was performed on 10-week-old WT female C57BL/6J mice. Three days post surgery, the OVX mice were intravenously injected with

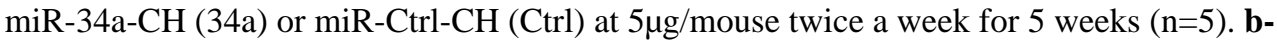
d, Osteoprotective effects of miR-34a-CH in sham control mice. WT female C57B/6J mice ( $\mathrm{n}=5,10$ week old) were subjected to sham operation and then treated with miR-34a-CH or miR-34a-Ctrl at $5 \mu \mathrm{g} /$ mouse twice a week for 5 weeks. b, Serum CTX-1. c, Serum P1NP. d, $\mathrm{BV} / \mathrm{TV}$ of proximal tibiae by $\mu \mathrm{CT}$. e, Histomorphometry of the distal femur and vertebrae in WT and 34a-Tie2-Tg mice after OVX. 34a-Tie2-Tg mice or controls (3-month-old, female, $\mathrm{n}=7$ ) were subjected to OVX or sham operation and analyzed 5 weeks post-surgery. 
a

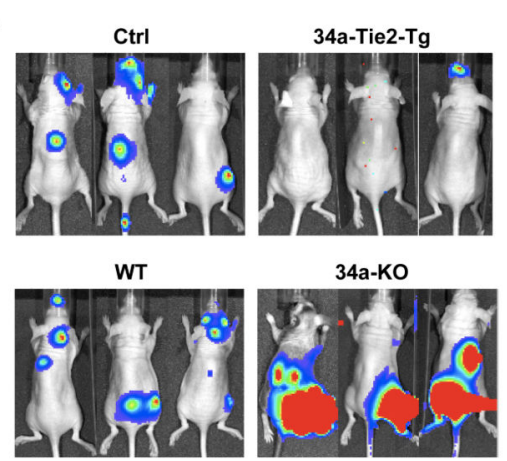

b

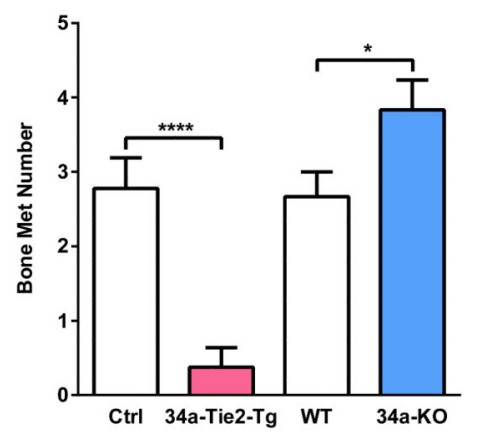

d
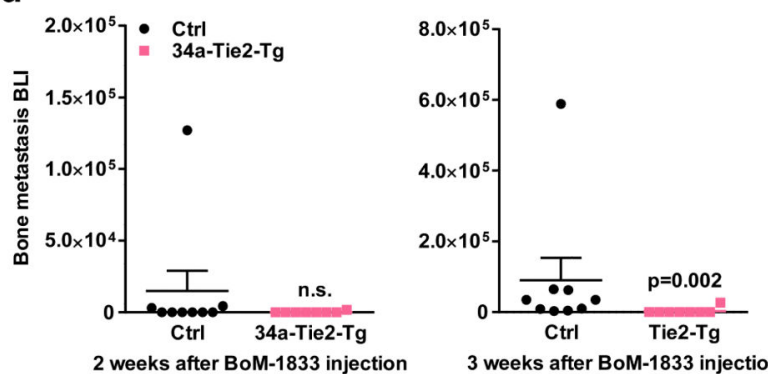

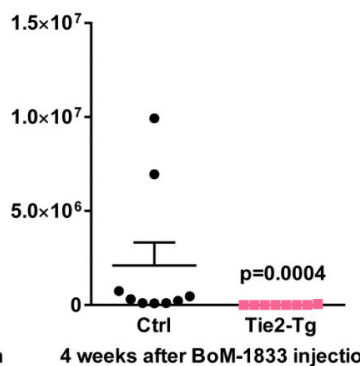

C
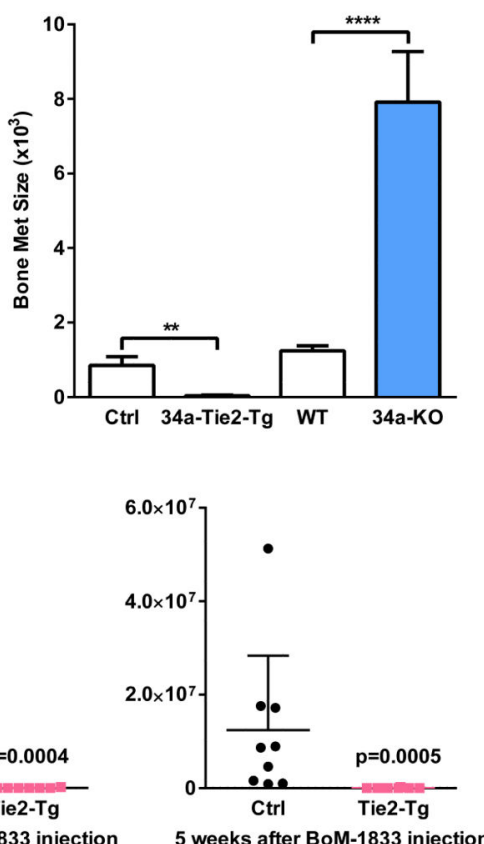

e
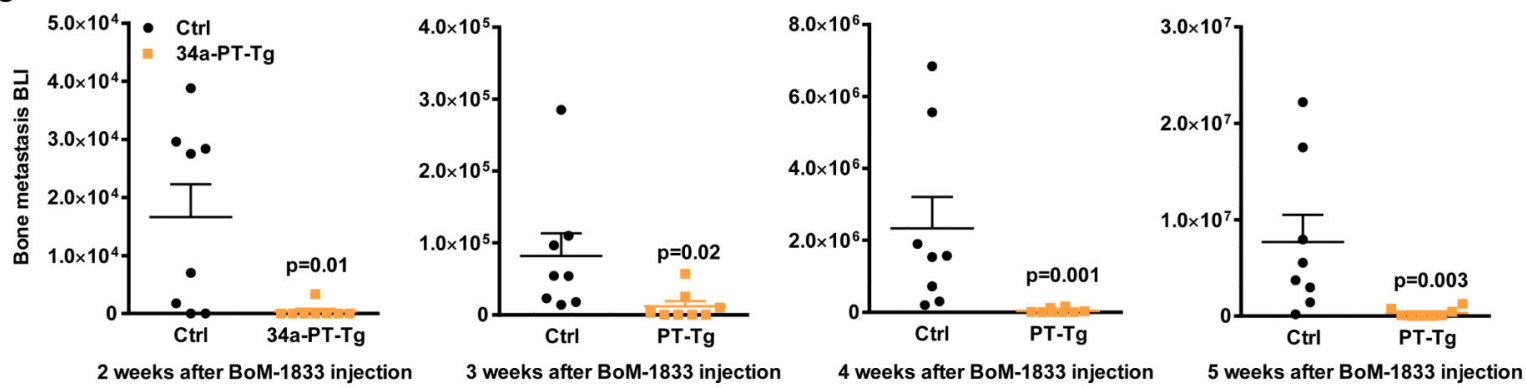

f

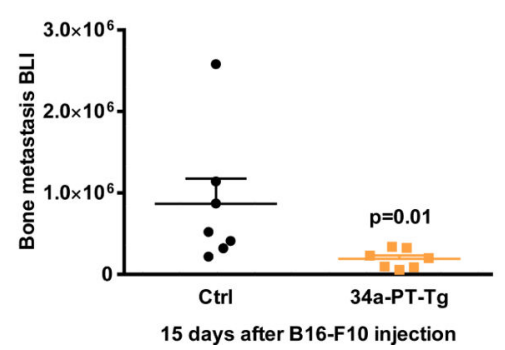

Extended Data Figure 7. Additional characterization of bone metastases

a, Representative BLI images. b, Quantification of the number of metastasis. c, Quantification of the size of metastasis. For a-c, $n=9$ for Ctrl, $n=8$ for $34 \mathrm{a}-\mathrm{Tie} 2-\mathrm{Tg}, \mathrm{n}=6$ for WT and 34a-KO; results are shown as average \pm s.e.. d, Xenograft of MDA231-BoM-1833 human breast cancer cells into 34a-Tie2-Tg nude mice $(n=8)$ or littermate control nude mice $(n=9)$. Results from each week are shown separately to better visualize the difference. e, Xenograft of MDA231-BoM-1833 human breast cancer cells into 34a-PT-Tg nude mice $(n=8)$ or littermate control nude mice $(n=8)$. Results from each week are shown separately to 
better visualize the difference. f, Allograft of B16-F10 mouse melanoma cells into 34a-PT$\operatorname{Tg}(n=7)$ or littermate control mice $(n=7)$. 
a
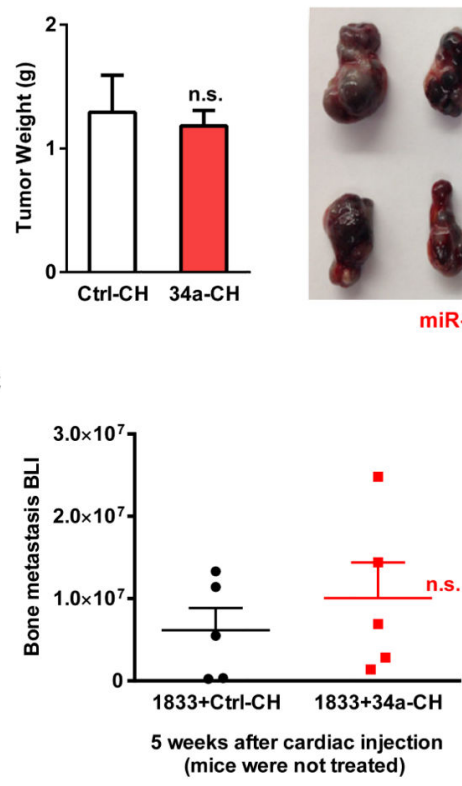

miR-Ctrl-CH

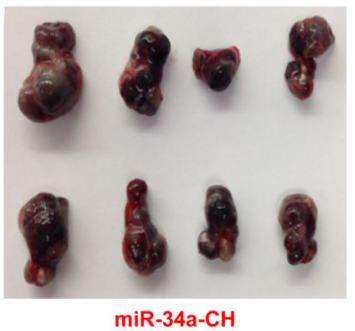

b
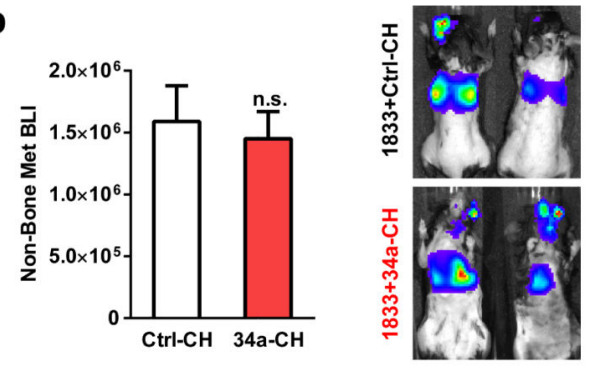

d

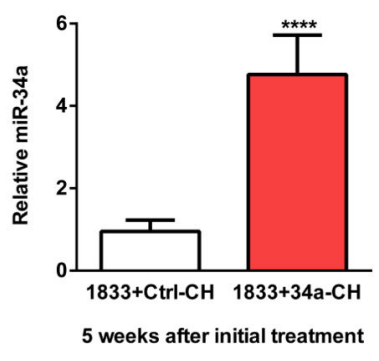

miR-34a $a^{+/-} \times$miR-34a $a^{+/-}$

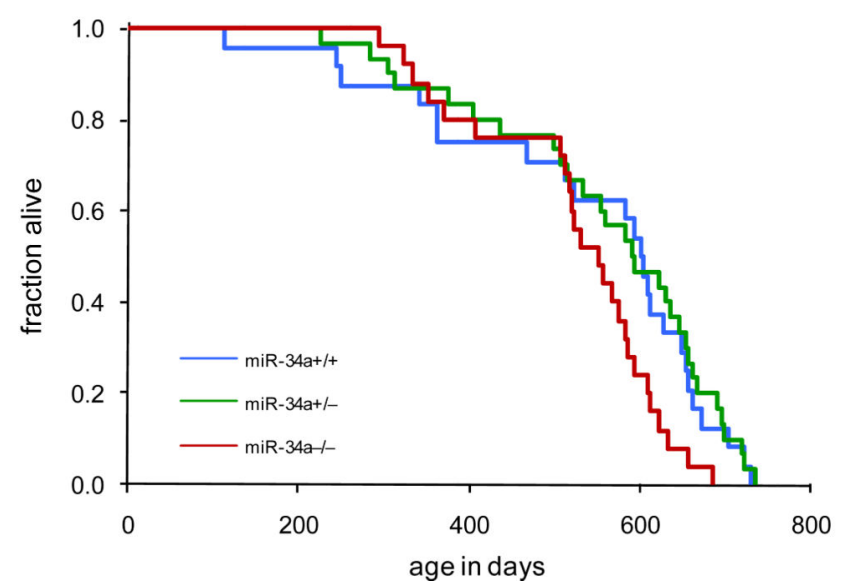

Extended Data Figure 8. Effects of miR-34a on cancer cells

a, Systemic miR-34a-CH delivery did not affect the growth of B16-F10 melanoma cells injected subcutaneously ( $n=5$, male, 8 week old). Tumors were collected 18 days after cell injection, result is shown as average \pm s.e. b, Systemic miR-34a-CH delivery did not affect cancer metastasis to other organs such as lung ( $n=5$, male, 8 week old). B16-F10 cells were i.v. injected retro-orbitally, BLI signals were quantified 2 weeks later and the result is shown as average \pm s.e. $\mathbf{c}-\mathbf{d}$, MiR-34a-CH treatment of cancer cell alone was not sufficient to inhibit bone metastasis. BoM-1833 cells were treated with miR-34a-CH or miR-Ctrl-CH in cultures for $24 \mathrm{hrs}$ before cardiac injection ( $\mathrm{n}=5$, male, 6 week old), and the mice were not treated with nanoparticles. c, Quantification of bone metastasis BLI signal 5 weeks after injection, shown as average \pm s.e. d, MiR-34a over-expression in BoM-1833 cells persisted for 5 weeks in cultures. e, Loss-of-function in 34a-KO and 34a-Het mice did not result in significantly increased susceptibility of cancer and mortality. Left, Kaplan-Meier survival 
curve for WT ( $\mathrm{n}=29), 34 \mathrm{a}-\mathrm{Het}(\mathrm{n}=35)$ and 34a-KO $(\mathrm{n}=29) ; \mathrm{p}=0.223$ by log-Rank (MantelCox) test. Right, the 34a-KO allele was transmitted at normal Mendelian frequency. 
a

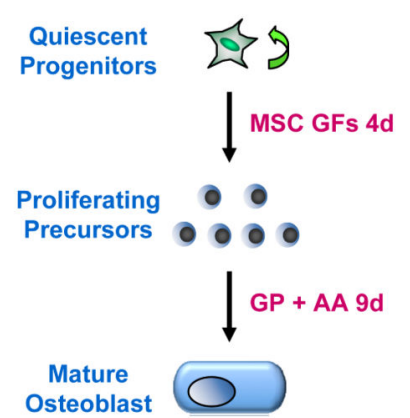

b

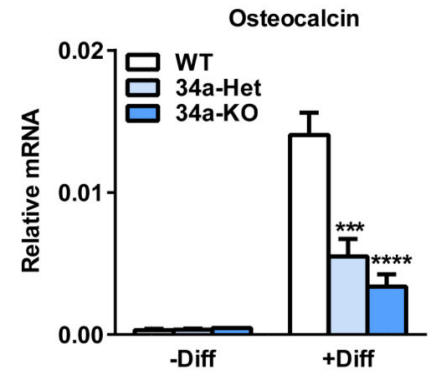

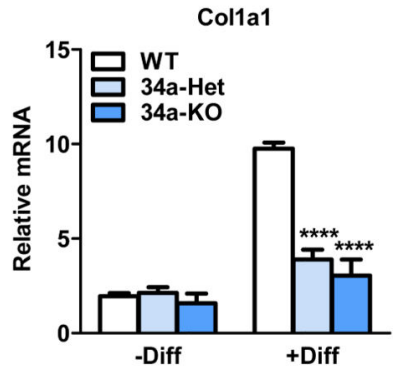

C

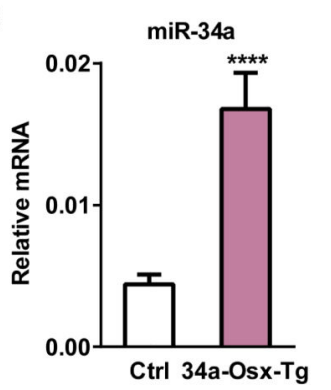

\begin{tabular}{|c|c|c|c|c|c|}
\hline & \multirow[b]{2}{*}{ Parameters } & \multicolumn{2}{|c|}{ Male } & \multicolumn{2}{|c|}{ Female } \\
\hline & & $\begin{array}{l}\mathrm{Ctrl} \\
(\mathrm{n}=6)\end{array}$ & $\begin{array}{c}34 a-O s x-T g \\
(n=6)\end{array}$ & $\begin{array}{c}\mathrm{Ctrl} \\
(\mathrm{n}=6)\end{array}$ & $\begin{array}{c}34 a-0 s x-T g \\
(n=5)\end{array}$ \\
\hline \multirow{7}{*}{ 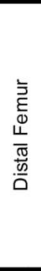 } & BV/TV (\%) & $15.37 \pm 0.71$ & $16.81 \pm 1.02^{*}$ & $13.96 \pm 0.91$ & $15.77 \pm 0.58^{*}$ \\
\hline & Oc.S/B.S (\%) & $5.21 \pm 0.38$ & $5.95 \pm 0.54$ & $5.56 \pm 0.24$ & $5.82 \pm 0.30$ \\
\hline & Oc.N/B.Ar (\%) & $5.16 \pm 0.52$ & $5.77 \pm 0.75$ & $5.25 \pm 0.31$ & $5.60 \pm 0.57$ \\
\hline & Ob.S/B.S (\%) & $6.22 \pm 0.15$ & $8.16 \pm 0.42^{\star}$ & $5.79 \pm 0.33$ & $7.65 \pm 0.29^{*}$ \\
\hline & Ob.N/B.Ar (\%) & $7.23 \pm 0.34$ & $9.39 \pm 0.42^{\star}$ & $6.85 \pm 0.47$ & $8.79 \pm 0.25^{*}$ \\
\hline & BFR/BS $\left(\mu \mathrm{m}^{3} / \mu \mathrm{m}^{2} /\right.$ year $)$ & $212.20 \pm 8.74$ & $266.29 \pm 10.38^{*}$ & $205.18 \pm 12.60$ & $251.63 \pm 9.67^{*}$ \\
\hline & $\operatorname{MAR}(\mu \mathrm{m} /$ day $)$ & $2.49 \pm 0.17$ & $2.91 \pm 0.08^{*}$ & $2.16 \pm 0.19$ & $2.59 \pm 0.12^{\star}$ \\
\hline \multirow{7}{*}{ 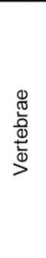 } & BV/TV $(\%)$ & $17.88 \pm 0.52$ & $19.54 \pm 0.66^{\star \star}$ & $16.26 \pm 0.83$ & $17.92 \pm 0.95^{\star}$ \\
\hline & Oc.S/B.S (\%) & $5.03 \pm 0.22$ & $5.28 \pm 0.36$ & $5.75 \pm 0.33$ & $6.07 \pm 0.59$ \\
\hline & Oc.N/B.Ar (\%) & $6.12 \pm 0.40$ & $6.32 \pm 0.56$ & $5.42 \pm 0.50$ & $5.85 \pm 0.42$ \\
\hline & Ob.S/B.S (\%) & $7.03 \pm 0.22$ & $8.98 \pm 0.39^{*}$ & $6.38 \pm 0.35$ & $8.22 \pm 0.52^{\star}$ \\
\hline & Ob.N/B.Ar (\%) & $8.30 \pm 0.51$ & $10.68 \pm 0.75^{*}$ & $7.75 \pm 0.39$ & $9.81 \pm 0.60^{*}$ \\
\hline & BFR/BS $\left(\mu \mathrm{m}^{3} / \mu \mathrm{m}^{2} /\right.$ year $)$ & $233.68 \pm 16.82$ & $285.09 \pm 12.75^{*}$ & $225.61 \pm 12.50$ & $271.55 \pm 14.73^{*}$ \\
\hline & $\operatorname{MAR}(\mu \mathrm{m} /$ day $)$ & $2.62 \pm 0.11$ & $3.02 \pm 0.16^{*}$ & $2.53 \pm 0.12$ & $2.96 \pm 0.15^{*}$ \\
\hline
\end{tabular}

d

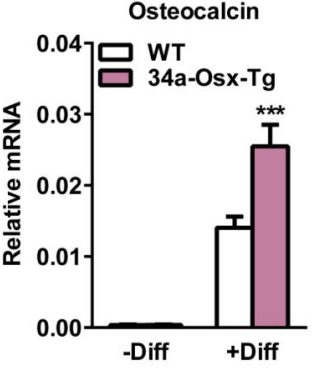

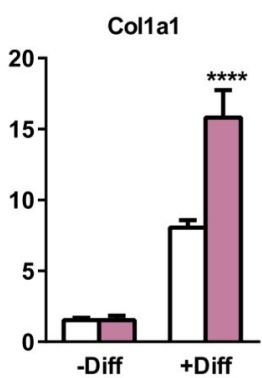

e

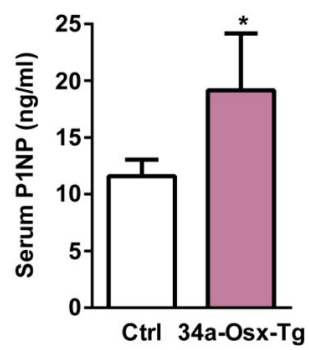

f

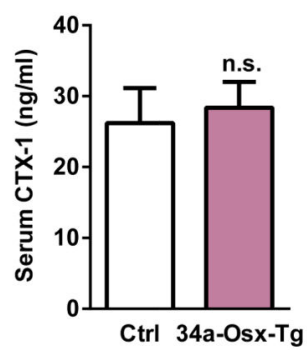

g

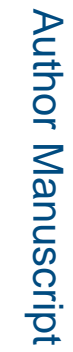

Extended Data Figure 9. Osteoblastic miR-34a over-expression is not sufficient to inhibit osteoporosis or bone metastases

a, A schematic diagram of the ex vivo bone marrow osteoblast differentiation assay. MSC GF, mesenchymal stem cell growth factors; GP, $\beta$-glycerophosphate; AA, ascorbic acid. b, Osteoblast differentiation was decreased for bone marrow from 34a-KO and 34a-Het mice compared to WT controls, quantified by osteoblast marker genes osteocalcin and Col1a1 on day $13(n=6)$. c-h, Characterization of osteoblastic miR-34a transgenic mice. CAG34a mice were bred with Osterix-CreER mice to generate miR34a-Osx-transgenic (34a-Osx-Tg) mice or littermate control mice that carry only CAG34a transgene; all mice (1-month-old, male) 
received tamoxifen injection on two consecutive days and analyzed 2 months later. c, Elevated levels of mature miR-34a in 34a-Osx-Tg osteoblast differentiation cultures on day 13 ( $n=6)$. d, Osteoblast differentiation was increased for bone marrow from 34a-Osx-Tg mice compared to control mice, quantified by osteoblast marker genes osteocalcin and Colla1 on day $13(n=6)$. e, Serum P1NP was increased in 34a-Osx-Tg mice $(n=6)$. $\mathbf{f}$, Serum CTX-1 was unaltered in 34a-Osx-Tg mice $(n=6)$. g, Histomorphometry of distal femur and vertebrae in 34a-Osx-Tg and control mice. $\mathbf{h}$, OVX-induced bone resorption and bone loss was unaltered in 34a-Osx-Tg mice. 34a-Osx-Tg mice or controls (3-month-old and 2 months after tamoxifen injection, female, $n=5$ ) were subjected to OVX or sham operation and analyzed 5 weeks post-surgery. i, Cancer bone metastasis was unaltered in 34a-Osx-Tg mice $(\mathrm{n}=8)$. Statistical analyses in $\mathbf{i}$ were performed with Mann Whitney Test and are shown as mean \pm standard error. 
a

\begin{tabular}{|c|c|c|c|c|}
\hline Gene symbol & Gene name & $\begin{array}{l}\text { Representative } \\
\text { transcript }\end{array}$ & $\begin{array}{l}\text { mRNA down-regulated by } \\
\text { pre-miR-34a }\end{array}$ & $\begin{array}{l}\text { 3'-UTR-Luc down-regulated } \\
\text { by pre-miR-34a }\end{array}$ \\
\hline TGIF2 & TGFB-induced factor homeobox 2 & NM_001199513 & ل & $\sqrt{ }$ \\
\hline Calcr & calcitonin receptor & NM_001164737 & $\sqrt{ }$ & $x$ \\
\hline LEF1 & lymphoid enhancer-binding factor 1 & NM_001130713 & $\sqrt{ }$ & $x$ \\
\hline YY1 & YY1 transcription factor & NM_003403 & $\sqrt{ }$ & $x$ \\
\hline ENG & endoglin & NM_000118 & $\sqrt{ }$ & $\times$ \\
\hline SYT1 & synaptotagmin I & NM_001135805 & $\times$ & N.D. \\
\hline PLCG1 & phospholipase C, gamma 1 & NM_002660 & $x$ & N.D. \\
\hline MAP2K1 & mitogen-activated protein kinase kinase 1 & NM_002755 & $\times$ & N.D. \\
\hline TFRC & transferrin receptor $(p 90, C D 71)$ & NM_001128148 & $\times$ & N.D. \\
\hline CSF1R & colony stimulating factor 1 receptor & NM_005211 & $x$ & N.D. \\
\hline STAT6 & $\begin{array}{l}\text { signal transducer and activator of transcription } \\
6 \text {, interleukin-4 induced }\end{array}$ & NM_001178078 & $x$ & N.D. \\
\hline IGFBP3 & insulin-like growth factor binding protein 3 & NM_000598 & $\times$ & N.D. \\
\hline SMAD4 & SMAD family member 4 & NM_005359 & $\times$ & N.D. \\
\hline CRHR1 & corticotropin releasing hormone receptor 1 & NM_001145146 & very low expression & N.D. \\
\hline
\end{tabular}

b

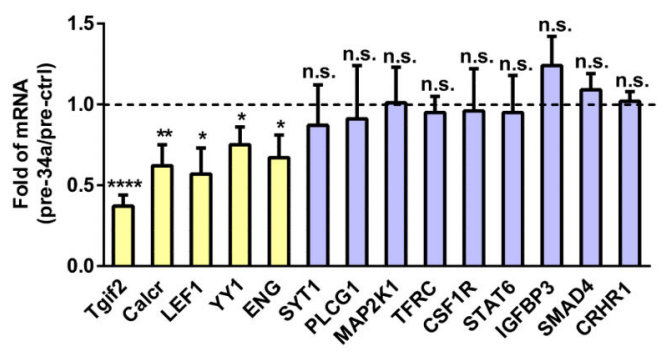

d

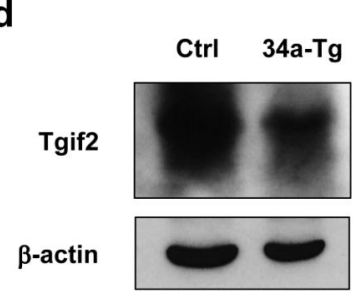

C

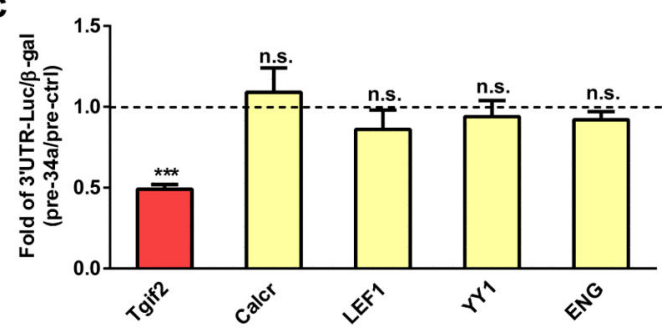

e

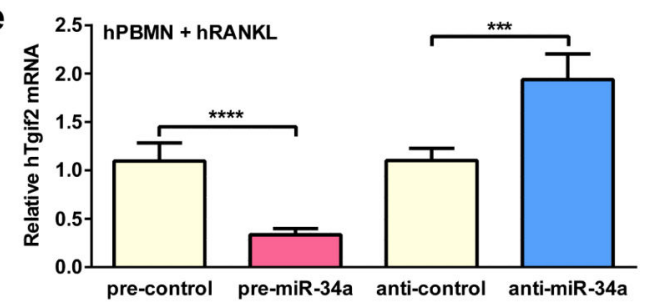

\begin{tabular}{|c|c|c|c|c|c|c|c|}
\hline & \multicolumn{3}{|c|}{ Male } & \multicolumn{3}{|c|}{ Female } \\
\hline & & WT & Tgif2-Het & Tgif2-KO & WT & Tgif2-Het & Tgif2-KO \\
\hline & Parameters & $(n=9)$ & $(n=10)$ & $(n=9)$ & $(n=6)$ & $(n=8)$ & $(n=7)$ \\
\hline \multirow{7}{*}{ 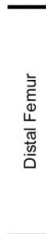 } & BV/TV (\%) & $15.86 \pm 1.24$ & $20.92 \pm 2.34^{* *}$ & $23.70 \pm 2.88^{t * \star}$ & $14.15 \pm 0.82$ & $19.32 \pm 2.86^{*}$ & $22.07 \pm 2.23^{* \star *}$ \\
\hline & Oc.S/B.S (\%) & $5.92 \pm 0.16$ & $3.14 \pm 0.05^{* *}$ & $2.23 \pm 0.04^{4 * * *}$ & $6.15 \pm 0.32$ & $3.25 \pm 0.21^{*}$ & $2.29 \pm 0.18^{* *}$ \\
\hline & Oc.N/B.Ar (\%) & $6.26 \pm 0.20$ & $3.02 \pm 0.19^{\text {t**}}$ & $2.18 \pm 0.25^{* * *}$ & $6.77 \pm 0.38$ & $3.26 \pm 0.15^{* *}$ & $2.45 \pm 0.12^{* * x}$ \\
\hline & Ob.S/B.S (\%) & $6.27 \pm 0.11$ & $6.13 \pm 0.13$ & $6.58 \pm 0.20$ & $5.92 \pm 0.24$ & $5.86 \pm 0.23$ & $5.84 \pm 0.38$ \\
\hline & Ob.N/B.Ar (\%) & $8.16 \pm 0.48$ & $8.23 \pm 0.59$ & $8.38 \pm 0.32$ & $7.67 \pm 0.52$ & $7.60 \pm 0.38$ & $7.71 \pm 0.69$ \\
\hline & $\operatorname{BFR} / \mathrm{BS}\left(\mathrm{um}^{3} / \mathrm{\mu m}^{2} /\right.$ year) & $192.67 \pm 6.45$ & $198.97 \pm 12.82$ & $199.14 \pm 8.96$ & $184.26 \pm 12.63$ & $182.05 \pm 15.10$ & $190.78 \pm 13.62$ \\
\hline & $\operatorname{MAR}(\mu \mathrm{m} / \mathrm{day})$ & $2.18 \pm 0.15$ & $2.21 \pm 0.20$ & $2.25 \pm 0.19$ & $1.93 \pm 0.16$ & $2.04 \pm 0.12$ & $2.07 \pm 0.21$ \\
\hline \multirow{7}{*}{ 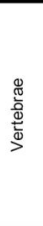 } & BVITV (\%) & $17.22 \pm 1.15$ & $22.86 \pm 1.98^{* *}$ & $25.38 \pm 2.07^{* * *}$ & $16.33 \pm 0.79$ & $21.34 \pm 1.33^{3^{* *+}}$ & $24.27 \pm 2.88^{* \star *}$ \\
\hline & Oc.S/B.S (\%) & $5.65 \pm 0.25$ & $3.16 \pm 0.11^{\star * *}$ & $2.15 \pm 0.07 * * * x$ & $5.96 \pm 0.22$ & $3.28 \pm 0.13^{* *}$ & $2.16 \pm 0.25^{* *}$ \\
\hline & Oc.N/B.Ar (\%) & $5.97 \pm 0.45$ & $2.86 \pm 0.12^{2 * *}$ & $1.92 \pm 0.22^{* *}$ & $6.55 \pm 0.37$ & $3.15 \pm 0.22^{* *}$ & $2.53 \pm 0.16^{* * * *}$ \\
\hline & Ob.S/B.S (\%) & $6.88 \pm 0.34$ & $6.93 \pm 0.51$ & $6.96 \pm 0.47$ & $6.63 \pm 0.27$ & $6.69 \pm 0.59$ & $6.72 \pm 0.45$ \\
\hline & Ob.N/B.Ar (\%) & $9.24 \pm 0.53$ & $9.30 \pm 0.67$ & $9.32 \pm 0.59$ & $8.85 \pm 0.66$ & $8.83 \pm 0.75$ & $8.92 \pm 0.48$ \\
\hline & BFR/BS $\left(\mu \mathrm{m}^{3} / \mathrm{m}^{2} /\right.$ year) & $203.91 \pm 8.68$ & $205.75 \pm 10.45$ & $204.86 \pm 9.64$ & $198.22 \pm 10.68$ & $199.06 \pm 15.41$ & $201.37 \pm 12.69$ \\
\hline & $\operatorname{MAR}(\mu \mathrm{m} /$ day) & $2.25 \pm 0.11$ & $2.24 \pm 0.16$ & $2.27 \pm 0.12$ & $2.22 \pm 0.17$ & $2.23 \pm 0.12$ & $2.25 \pm 0.15$ \\
\hline
\end{tabular}

Extended Data Figure 10. Additional characterization of Tgif2 as a key miR-34a direct target gene

a, A list of potential miR-34a target genes in the osteoclast lineage and characterization of miR-34a regulation. N.D., not determined. b, Fold changes in the expression of each candidate target gene after transfection with pre-miR-34a vs. pre-miR-ctrl in WT bone marrow osteoclast differentiation culture $(n=3)$. $\mathbf{c}$, Fold changes in the luciferase readout from 3'UTR reporter for each candidate target gene co-transfected in HEK293 cells with pre-miR-34a or pre-control. The results were normalized by internal control $\beta$-galactosidase 
( $\beta$-gal) readout $(\mathrm{n}=3)$. $\mathbf{d}$, Western blot analysis showing that Tgif2 protein expression is decreased in the bone marrow osteoclast progenitors from 34a-Tie2-Tg transgenic mice compared with control mice (left), but increased in the bone marrow osteoclast progenitors from 34a-KO and 34a-Het mice compared with WT control mice (right). e, Human Tgif2 expression in $\mathrm{hPBMN}$ osteoclast differentiation cultures was suppressed by pre-miR-34a but enhanced by anti-miR-34a via transfection $(n=4)$. $\mathbf{f}$, Histomorphometry of the distal femur and vertebrae in 1.5 month old Tgif2-KO, Tgif2-Het and WT control mice. 